\title{
Cellular Communications Using Aerial Platforms
}

\author{
Bashir El-Jabu and Raymond Steele, Fellow, IEEE
}

\begin{abstract}
This paper is devoted to the study of cellular communications using aerial platforms (APs). A set of key equations is derived that quantify the coverage area on the ground as a function of AP elevation, the operation of the adaptive multibeam antenna on the AP, and the formulation of contiguous terrestrial cells and their shapes. Specifically, we consider the deployment of an AP to provide terrestrial mobile radio communications using the universal mobile telecommunication system operating in its wide-band code-division multiple-access mode. Calculations are made of the number of users versus $E_{b} / N_{0}$ for different service rates. Multitiered cellular structures having cells of different size that are steerable with the offered teletraffic are examined. The array structure to achieve this is identified. The preliminary results shows that an $A P$ at a height of $21 \mathrm{~km}$ covers an area of radius $517 \mathrm{~km}$. Up to 21 users per cell with a service rate of $8 \mathrm{~kb} / \mathrm{s}$ can be accommodated in the 2.2-GHz band. These services can be provided within an area of radius $70 \mathrm{~km}$ with transmitted powers of less than $1 \mathrm{~W}$. High system capacity is proved to be possible by constructing cells of radius as small as $100 \mathrm{~m}$ using square planar arrays with dimensions of less than $12 \mathrm{~m} \times 12 \mathrm{~m}$. The AP system provides high capacity and Doppler frequency shifts that only originate from roving mobiles.
\end{abstract}

Index Terms-Aerial platforms, cellular communications, code division multiple access (CDMA).

\section{INTRODUCTION}

W ORLD-WIDE telephony and telegraphy supporting communications between fixed points has been available for many decades. Today, people want at their disposal a wide range of multimedia services that they can use while on the move, or when stationary but not near a tethered terminal. Further, the number of people requiring mobile services is growing at an exponential rate. Consequently, networks in the twenty-first century will need to respond to rapid demands in offered traffic that are difficult to predict with regard to time and geography.

The conventional way of designing a mobile network is to form cells of different shapes. This requires the acquisition of cell sites and the deployment of base-station (BS) equipment. Not only are BSs unable to respond to massive peaks in offered traffic, but they often spend a large fraction of the day in an idle state. For example, BSs in a business district may be very busy for ten hours a day, but when the business people depart, there is negligible offered traffic. The BS capacity cannot be switched elsewhere, such as to suburbia, where it is likely to be required. We therefore have a double problem. We do not know when or

Manuscript received September 4, 1998; revised July 19, 2000. This work was supported by the Secretariat of Education, Libya.

B. El-Jabu is with the Electrical Engineering Department, Al-Fateh University, Libya, and the Higher Industrial Institute, Misurata, Libya.

R. Steele is with the Department of Electronics and Computer Science, University of Southampton, Southampton SO17 1BJ, U.K.

Publisher Item Identifier S 0018-9545(01)03963-9. where peaks of traffic may occur, and the network is unable to adapt its offered capacity according to demand. To make matters worse, fixed cell sites may incur high rental charges. As capacity requirements rise, so must the amount of infrastructure.

To solve this problem, we need a different approach. In 1992, Steele proposed the use of stationary aerial platforms to handle moving peaks of teletraffic: "The platforms will be tethered to the earth and located up to $30 \mathrm{~km}$ in height and placed between the aircraft flying lanes. Barely visible from the earth they will be able to deliver many services. They will be held on station by power conveyed to them via their tie-lines, and these lines will also house the fibers that convey the teletraffic with the network. Alternatively these platforms could be untethered, hovering, and therefore capable of being rapidly re-deployed. The hovering platforms would communicate to earth via radio. The tethered or hovering platforms will be able to track 'solitons of teletraffic,' rather than force the task on to the terrestrial networks. For example, the platforms could handle the teletraffic from high speed trains, highways, aircraft and ships. They can be rapidly deployed when disasters occur, for example, the rapid provision of communications to a city which has been devastated by an earthquake" [1]. Later, the application of aerial platforms for cellular networks was described in an editorial [2].

Since then, much has happened. Unmanned sky platforms have been proposed. The platforms will consist of a multilayer skin airship having buoyant helium-filled cells, a station-keeping system, solar arrays for daytime power, and fuel cells for nighttime power. They will utilize a global positioning system for accurate position measurement, and ultrathin fabric hulls for long duration buoyancy [3].

The deployment of aerial platforms (APs) using multiple-beam phased array technology offers the prospect of deploying the network capacity when and where it is needed. Located at elevations of a few to $25 \mathrm{~km}$ or so, the APs are stationary and therefore do not contribute to Doppler frequency shift in radio transmissions. APs are sufficiently close to the earth that their path loss is low and compatible with those in conventional urban macrocells due to the free-space loss once the transmissions clear the city skyline. The capacity of APs can be high, as they are able to form small cells on the earth's surface, as we will describe later, and have a good reuse factor. The APs can be moved to where they are required, when their elevation can be adjusted to suit network requirements. Moreover, they can be interconnected to form sky networks where APs are not only networked access nodes but also act as repeaters. Although an AP may form small cells by the use of multiple beams, they can also form megacells that span conventional terrestrial cellular structures [1].

This paper is concerned with the basic equations relating to aerial platform communications. Accordingly, we assume that 
an AP is stationary, although we recognize that station-keeping is a far from trivial task. If an AP is an aircraft, such as the Proteus made by Angel Technologies, then it will fly in tight circles (some five to eight miles in diameter), with the station keeping about the center of the circle. We are not considering this type of AP in this paper. The airship type of AP must have sufficient propulsion to overcome stratospheric winds, which for a given location vary through the year. Even if sufficient propulsion is available, there is still the problem of combatting the small vertical, lateral, and tilting movements. Positional instability of APs has been studied [4], where it is shown that the tilt of an $\mathrm{AP}$ is the movement that has the most deleterious effect. If not restrained to less than $3^{\circ}$, it can significantly decrease the capacity of a code-division multiple-access (CDMA) AP network. However, as we are concerned here with basic equations and parameters, the stability issues will not be addressed.

\section{THE VIEW OF THE EARTH FROM THE AERIAL PlatFORM}

The extremities of the "view" of the earth's surface from the aerial platform at point $\mathrm{P}$ in Fig. 1 is found by drawing a straight line from $\mathrm{P}$ to make a tangent with the earth's surface at point $\mathrm{A}$, and then rotating the arc $\mathrm{AD}$ by $360^{\circ}$ about the $y$-axis. The surface area mapped out is

$$
S=2 \pi R_{e} W
$$

where $R_{e}$ is the radius of the earth $\left(R_{e} \cong 6370 \mathrm{~km}\right)$ and $W$ is the depth from the earth's surface, point $\mathrm{D}$, that is immediately below $\mathrm{P}$, to a plane within the earth surface that connects with points A and B. From triangle OAP, the distance from the platform to point $\mathrm{A}$ is

$$
L=\sqrt{h^{2}+2 R_{e} h}
$$

where $h$ is the nadir. From triangles FAP and OAF, we have

$$
L^{2}=r^{2}+(h+W)^{2}
$$

and

$$
R_{e}^{2}=r^{2}+\left(R_{e}-W\right)^{2}
$$

respectively, where $r$ is shown in Fig. 1. Eliminating $r^{2}$ from (3) and (4) and using (2) with $W$ obtained from (1) yields

$$
h=\frac{S}{2 \pi R_{e}-\frac{S}{R_{e}}} .
$$

For the situation where $S \ll 2 \pi R_{e}{ }^{2}$, i.e., $S<25 \times 10^{6}$ square kilometers, $h=S / 2 \pi R_{e}$. The variation of the radius of the total surface area covered by the aerial platform versus its height $h$ is displayed in Fig. 2.

The angle OPA in Fig. 1 is

$$
\theta=\cos ^{-1} \frac{L}{R_{e}+h}
$$

and the curve from $\mathrm{A}$ to $\mathrm{B}$ is

$$
\overline{A B}=2 R_{e} \tan ^{-1} \frac{L}{R_{e}} .
$$

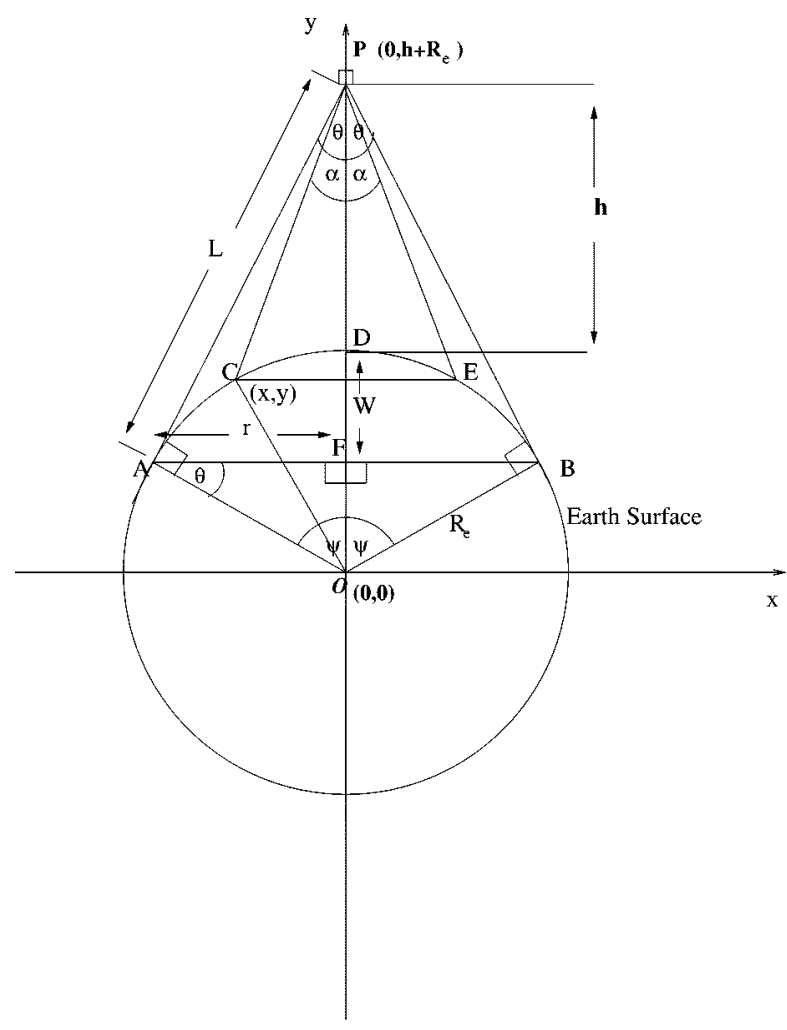

Fig. 1. Cross-section at $z=0$ in the space-earth geometry of the AP. The AP is at position $\mathrm{P}$.

The straight line $\mathrm{AB}$, which passes through point $\mathrm{F}$, is given by

$$
\mathrm{AB}=\frac{2 R_{e} \sqrt{h^{2}+2 R_{e} h}}{R_{e}+h} .
$$

To approximate the curve $\mathrm{AB}$ through $\mathrm{D}$ by the straight line $\mathrm{AB}$, the following condition should be satisfied:

$$
\tan ^{-1} \frac{\sqrt{h^{2}+2 R_{e} h}}{R_{e}} \cong \frac{\sqrt{h^{2}+2 R_{e} h}}{R_{e}+h} .
$$

Using the fact that $\tan (\theta) \cong \theta$ for $\theta<8^{\circ}$, (9) is valid if $h \ll R_{e}$, when

$$
\frac{\sqrt{h^{2}+2 R_{e} h}}{R_{e}}<\frac{8 \pi}{180}
$$

or $h<62 \mathrm{~km}$.

Consider point $\mathrm{C}$ on the earth's surface, having the coordinates $(x, y)$ referenced to the center of the earth, as shown in Fig. 1 . The distance from the platform to point $(x, y)$ is

$$
\mathrm{PC}=\sqrt{x^{2}+\left(h+R_{e}-y\right)^{2}}
$$

for $-r \leq x \leq r$ and $R_{e}-W \leq y \leq R_{e}$. Substituting from the previous equations for $x$ and $L$, the length PC becomes

$$
\mathrm{PC}=\sqrt{L^{2}+2 R_{e}^{2}-2 y\left(h+R_{e}\right)}
$$

and the arc $\mathrm{CD}$ is

$$
\mathrm{CD}=R_{e} \tan ^{-1} \frac{\sqrt{R_{e}^{2}-y^{2}}}{y} .
$$




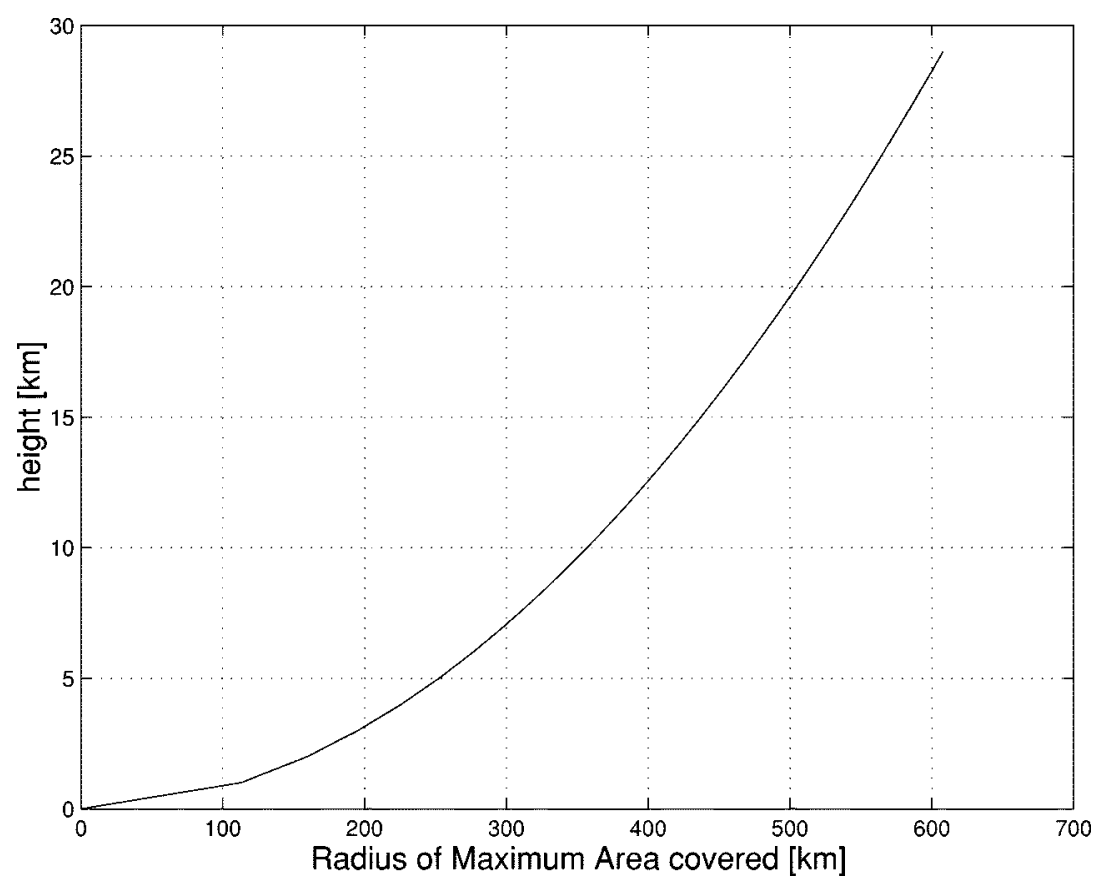

Fig. 2. The height of the AP versus the radius of the maximum area covered on the earth's surface.

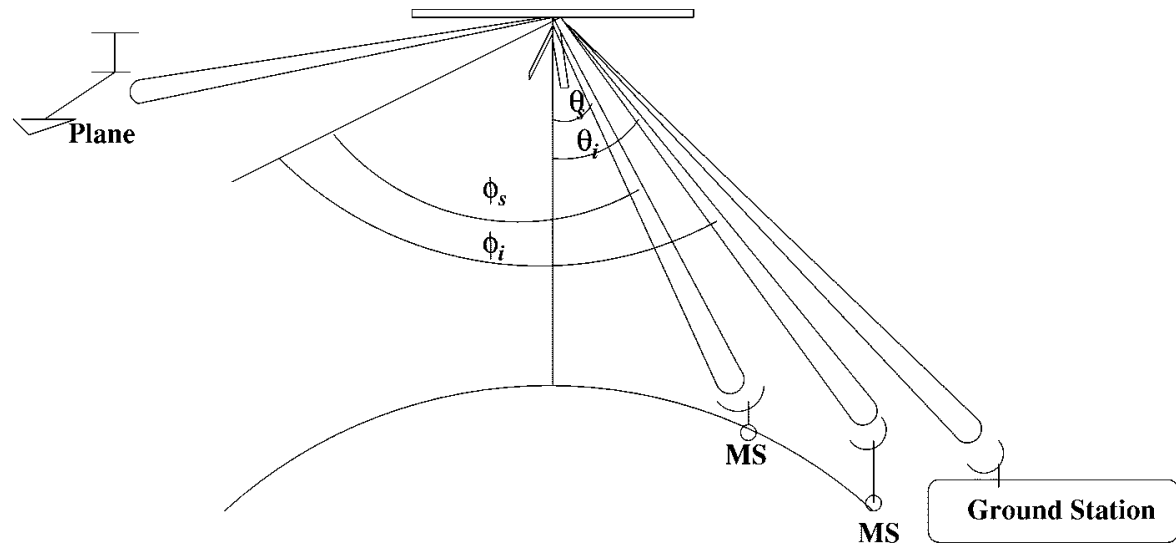

Fig. 3. Communications via multibeams in the AP system.

The total angle coverage of the platform $\left(\theta_{t}\right)$ is $2 \theta$ and can be evaluated from

$$
\theta_{t}=2 \cos ^{-1} \frac{\sqrt{2 R_{e} h+h^{2}}}{R_{e}+h} .
$$

The angle $\alpha$ in Fig. 1 is

$$
\alpha=\tan ^{-1} \frac{\sqrt{R_{e}^{2}-y^{2}}}{h+R_{e}-y}
$$

while the surface area containing $\mathrm{CDE}$ is

$$
S_{\mathrm{CE}}=2 \pi R_{e}\left(R_{e}-y\right)
$$

and

$$
y=R_{e}-\frac{S_{\mathrm{CE}}}{2 \pi R_{e}}
$$

\section{Multibeam AntennA}

The aerial platform will support a multibeam antenna. One beam will be directed at all times to the fixed network ground station that is connected to the cellular network. The other beams will be directed to fixed ground sites if they provide back-haul links from ground macrocellular or microcellular systems; or they will be steerable beams if they have to accommodate mobile traffic, which varies in location and intensity hour by hour. These steerable beams provide the network's capacity-on-demand feature. Fig. 3 shows a number of beams, including one that is tracking an airplane and offering the pilot and passengers communication links to a global network, e.g., to the Universal Mobile Telecommunication Services (UMTS) global network.

Each beam from the multibeam antenna can be steered independently. Different arrays can be used for this task; such as linear, circular, and planar arrays. Fig. 4 shows the arrangement of a linear adaptive array having $N$ elements with a distance 


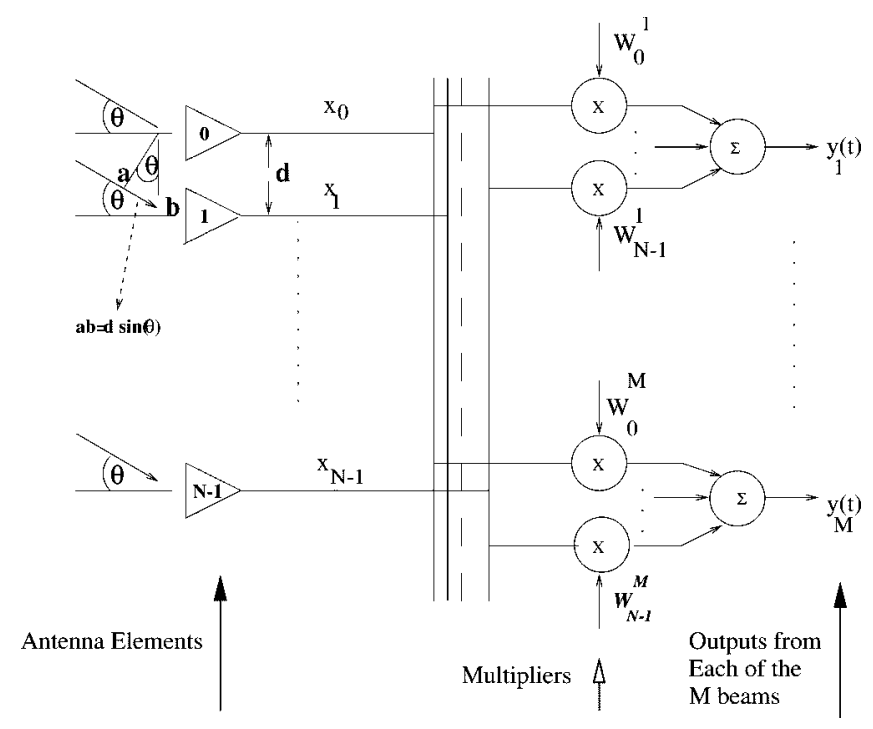

Fig. 4. Beamforming at the AP receiver.

$d$ between each two adjacent elements, which generates $M$ receiving beams.

Let us consider the reception of signals from the ground. Because the source is in the far field, the received rays are parallel. The differential distance along the two ray paths is $d \sin (\theta)$, where $\theta$ is an arbitrary angle of arrival $(-(\pi / 2) \leq \theta \leq(\pi / 2))$. Notice that the angle of arrival $\theta$ is different for each beam, and Fig. 4 displays the arrival of only one beam. Each element is weighted with a complex weight $W_{n}^{i}$, with $n=0,1, \ldots, N-1$ and $i=1,2, \ldots, M$. Assuming that the phase of the received ray at antenna element 0 is zero, the output of beam $i$ of the array is

$$
\begin{aligned}
y_{i}(t)= & W_{0}^{i} e^{j 2 \pi f_{0} t}+W_{1}^{i} e^{j 2 \pi f_{0}(t+\tau)} \\
& +\cdots+W_{N-1}^{i} e^{j 2 \pi f_{0}(t+(N-1) \tau)}
\end{aligned}
$$

where

$$
\tau=\frac{d \sin (\theta)}{c}
$$

and $c$ is the speed of light. Substituting $\tau$ from (19) into (18) yields

$$
y_{i}(t)=F_{i}(\theta) e^{j 2 \pi f_{0} t}
$$

where $\beta=2 \pi / \lambda, \lambda$ is the wavelength, and

$$
F_{i}(\theta)=\sum_{n=0}^{N-1} W_{n}^{i} e^{j \beta n d \sin (\theta)}
$$

is the array factor.

Let the phase of the $n$th element lead that of the $(n-1)$ th element by $\alpha_{i} d$. On introducing a weight factor $A_{i}(n)$ for the $i$ th beam, element $n$, we have

$$
W_{n}^{i}=A_{i}(n) e^{i n \alpha_{i} d}
$$

Accordingly, the $i$ th array factor becomes

$$
F_{i}(\theta)=\sum_{n=0}^{N-1} A_{i}(n) e^{j\left(\beta n d \sin (\theta)+n \alpha_{i} d\right)} .
$$

The beam shape depends on the weights. Assuming a uniform distribution, i.e., all of the weights have the same magnitude, $A_{i}(n)=A_{i}$ for all $n$. Using the formula $\sum_{n=0}^{N-1} a^{n}=(1-$ $\left.a^{n} / 1-a\right)$ gives the array factor

$$
F_{i}(\theta)=A_{i} e^{j(N / 2)\left[\alpha_{i}+\beta \sin (\theta)\right] d} \frac{\left.\sin \left\{\frac{N}{2}\left[\alpha_{i}+\beta \sin (\theta)\right] d\right]\right\}}{\sin \left\{\left[\alpha_{i}+\beta \sin (\theta)\right] \frac{d}{2}\right\}}
$$

the absolute value of which is

$$
\left|F_{i}(\theta)\right|=A_{i}\left|\frac{\sin \left\{\frac{N}{2}\left[\alpha_{i}+\beta \sin (\theta)\right] d\right\}}{\sin \left\{\left[\alpha_{i}+\beta \sin (\theta)\right] \frac{d}{2}\right\}}\right| .
$$

For the $i$ th beam to be at an angle $\theta_{i}, \alpha_{i}=-\beta \sin \left(\theta_{i}\right)$, and the power pattern of this array in the direction $\theta_{i}$ becomes

$$
\left|F_{i}(\theta)\right|=A_{i}\left|\frac{\sin \left[\frac{\pi N d}{\lambda}\left\{\sin (\theta)-\sin \left(\theta_{i}\right)\right\}\right]}{\sin \left[\frac{\pi d}{\lambda}\left\{\sin (\theta)-\sin \left(\theta_{i}\right)\right\}\right]}\right|
$$

which has a maximum gain of $A_{i} N$.

By controlling the magnitude of the weights and the number of elements, the gain of each beam can be controlled, with each beam having $(N-2)$ side lobes. The phase of the weights $W_{n}^{i}$ are used to control the direction of the main beam. The nulls of the main beam are obtained from (26) when

$$
\frac{\pi N d}{\lambda}\left\{\sin (\theta)-\sin \left(\theta_{i}\right)\right\}= \pm \pi
$$

from which the width of the main beam between zeros is

$$
B W_{0}=\sin ^{-1}\left[\sin \left(\theta_{i}\right)+\frac{\lambda}{N d}\right]-\sin ^{-1}\left[\sin \left(\theta_{i}\right)-\frac{\lambda}{N d}\right] .
$$

Thus, the beamwidth depends on the number of elements, the distance between them (relative to the wavelength), and the direction of the beam. In this context, the following points are worth mentioning.

1) Equation (26) repeats every $2 \pi$ radians, and so more than one main beam in the visible region may occur. To avoid this, the spacing $d$ should always be kept less than $\lambda$.

2) For a given number of elements, increasing the spacing between them decreases the beamwidth and increases the array size $(N d)$.

As an example, consider a linear array of ten elements with $d=\lambda / 2$ used to generate three independent beams at angles $-30^{\circ}, 0^{\circ}$, and $60^{\circ}$. The resultant beams are shown in Fig. 5 and 


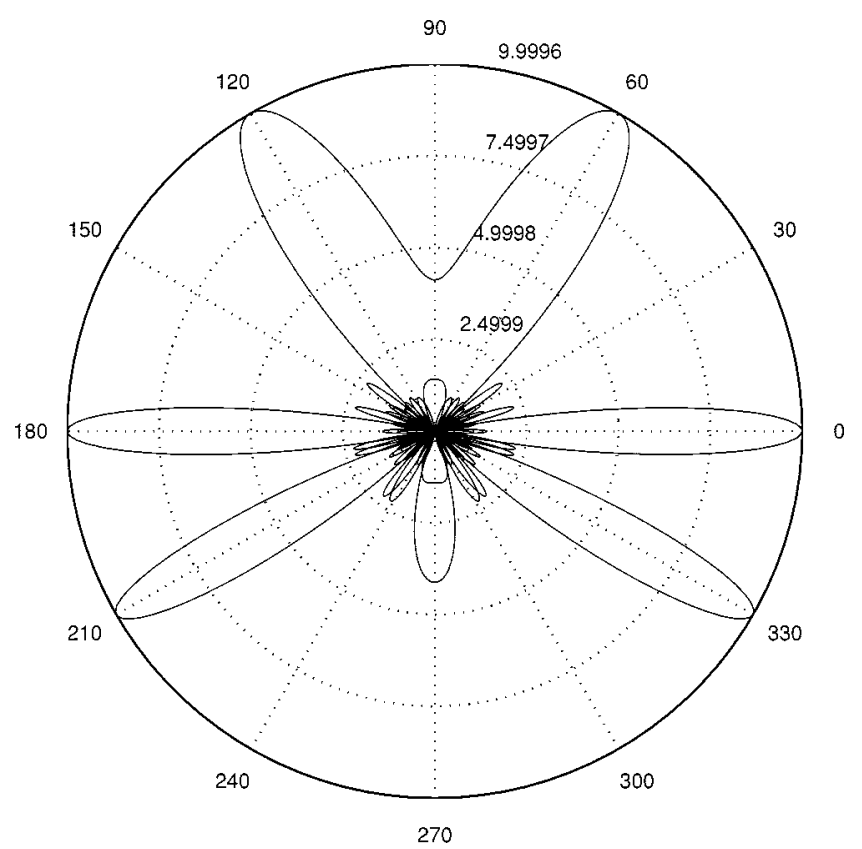

Fig. 5. Three beams at $-30^{\circ}, 0^{\circ}$, and $60^{\circ}$ formed by a ten-element linear array.

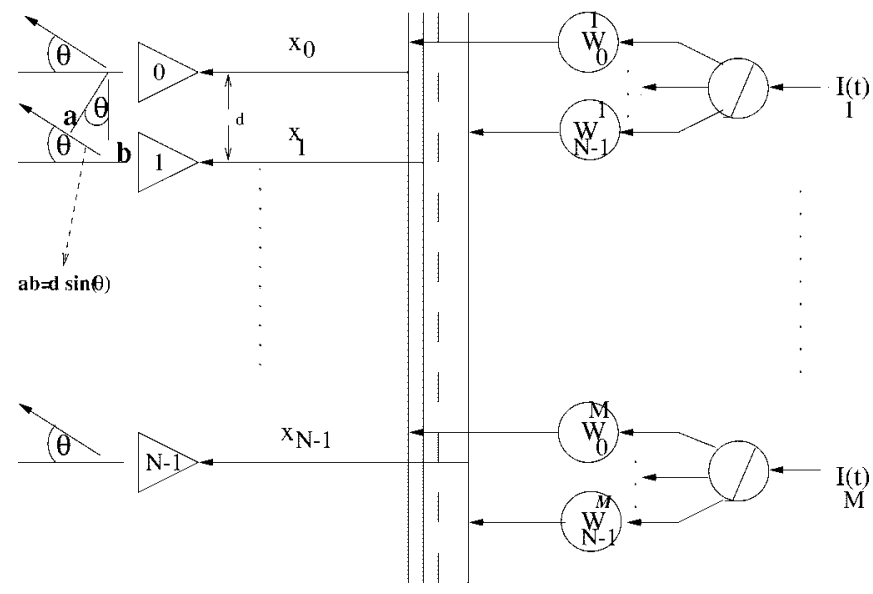

Fig. 6. Beamforming at the AP transmitter.

have different beamwidths. The patterns are symmetrical about the axis of the array.

Generally, the 3-dB beamwidth is obtained by solving (26) numerically, although there are some approximated formulas for special cases [5].

Let us now consider generating the multibeams for transmission. The weights now control the magnitude and the phase difference of the currents fed to the elements, as indicated in Fig. 6. If the weights are chosen such that $I_{n}^{i}=I_{i} e^{j n \alpha_{i} d}$, then the power pattern of the beam directed at an angle $\theta_{i}$ becomes

$$
\left|F_{i}(\theta)\right|=I_{i}\left|\frac{\sin \left[\frac{\pi N d}{\lambda}\left\{\sin (\theta)-\sin \left(\theta_{i}\right)\right\}\right]}{\sin \left[\frac{\pi d}{\lambda}\left\{\sin (\theta)-\sin \left(\theta_{i}\right)\right\}\right]}\right|
$$

which has a maximum gain of $I_{i} N$.

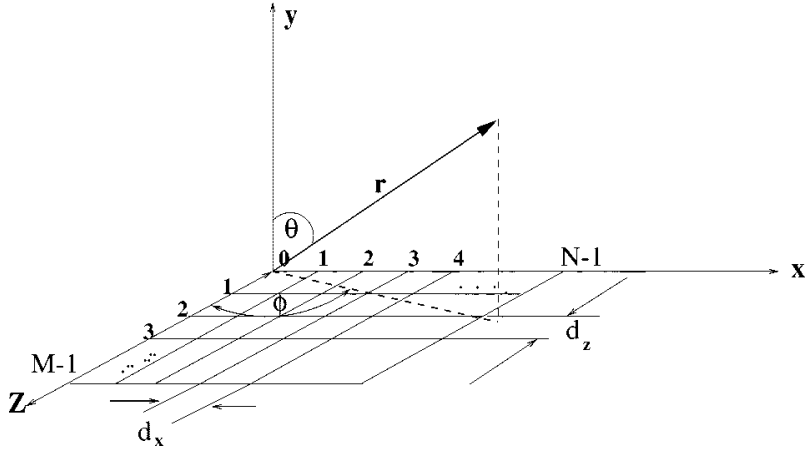

Fig. 7. Two-dimensional array of $N \times M$ elements.

\section{A. Two-Dimensional Arrays}

A rectangular or planar array consists of $N$ and $M$ elements along the $x$ and $y$ ordinates, respectively, as shown in Fig. 7 [5]. This array may be viewed as $M$ linear arrays, and the twodimensional (2-D) array factor is found by multiplying the array factor for the $M$ arrays along the $y$ axis by the array factor for the $N$ dipoles along the $x$ axis [6], i.e.,

$$
\begin{aligned}
& F_{1}(u)=\sum_{n=0}^{N-1} A_{n} e^{\left(j \beta n d_{x} \sin (u)+\beta_{x}\right)} \\
& F_{2}(v)=\sum_{m=0}^{M-1} B_{m} e^{\left(j \beta m d_{z} \sin (v)+\beta_{z}\right)}
\end{aligned}
$$

and

$$
F(u, v)=F_{1}(u) F_{2}(v)
$$

where $F(u, v)$ is the overall array factor with $\sin (u)=$ $\sin (\theta) \cos (\phi)$ and $\sin (v)=\sin (\theta) \sin (\phi)$. In (30) and (31), $\beta_{x}$ is the phase shift between the elements in the $x$-axis, while $\beta_{y}$ is the phase shift between the elements in the $y$-axis.

Repeating the steps of the one-dimensional case, and for a uniform array, the array factor of the 2-D array directed toward $\left(\theta_{i}, \phi_{k}\right)$ is

$$
\begin{aligned}
& \left|F_{i k}(\theta, \phi)\right| \\
& =C_{i k} \cdot\left|\frac{\sin \left[\frac{\pi N d_{x}}{\lambda}\left\{\sin (\theta) \cos (\phi)-\sin \left(\theta_{i}\right) \cos \left(\phi_{k}\right)\right\}\right]}{\sin \left[\frac{\pi d_{x}}{\lambda}\left\{\sin (\theta) \cos (\phi)-\sin \left(\theta_{i}\right) \cos \left(\phi_{k}\right)\right\}\right]}\right| \\
& \cdot\left|\frac{\sin \left[\frac{\pi M d_{y}}{\lambda}\left\{\sin (\theta) \sin (\phi)-\sin \left(\theta_{i}\right) \sin \left(\phi_{k}\right)\right\}\right]}{\sin \left[\frac{\pi d_{y}}{\lambda}\left\{\sin (\theta) \sin (\phi)-\sin \left(\theta_{i}\right) \sin \left(\phi_{k}\right)\right\}\right]}\right| .
\end{aligned}
$$

For a large array, where the beams are close to the broadside, the elevation plane half-power beamwidth (HPBW) is given approximately by [5]

$$
\mathrm{BW}_{\theta}=\frac{1}{\cos \left(\theta_{i}\right) \sqrt{\theta_{x 0}^{-2} \cos ^{2}\left(\phi_{k}\right)+\theta_{y 0}^{-2} \sin ^{2}\left(\phi_{k}\right)}}
$$




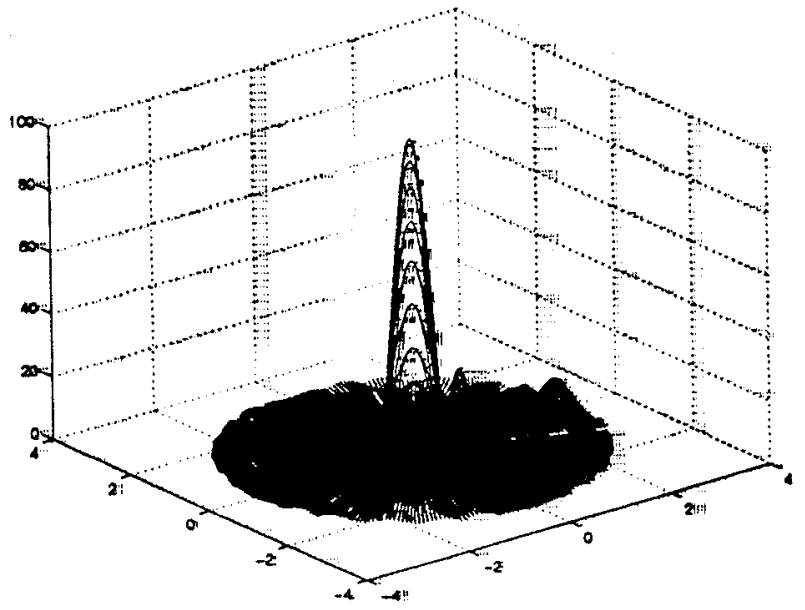

Fig. 8. Three-dimensional pattern for square array of $10 \times 10$ elements with spacing $d=\lambda / 2$ directed toward $(0,0)$.

where $\theta_{x 0}$ and $\theta_{y 0}$ represent the half-power bandwidths. For a square array $N=M ; d_{x}=d_{y}, \theta_{x 0}=\theta_{y 0}$; and

$$
B W_{\theta}=\theta_{x 0} \mathrm{~s}\left(\theta_{i}\right)=\theta_{y 0} \mathrm{~s}\left(\theta_{i}\right)
$$

For the plane that is perpendicular to the $\phi=\phi_{k}$ elevation, the half-power beamwidth is

$$
\mathrm{BW}_{\phi}=\frac{1}{\sqrt{\theta_{x 0}^{-2} \sin ^{2}\left(\phi_{k}\right)+\theta_{y 0}^{-2} \cos ^{2}\left(\phi_{k}\right)}}
$$

which reduces to

$$
\mathrm{BW}_{\phi}=\theta_{x 0}=\theta_{y 0}
$$

for a square array.

As an example, consider a square array of $10 \times 10$ elements where the distance between each two elements on the same row or the same column is $d=\lambda / 2$. This array produces a threedimensional pattern directed toward $(0,0)$, as shown in Fig. 8. However, this pattern may be modified to electronically steer the central beam as required. An identical pattern is formed in the lower hemisphere, which can be diminished by the use of a ground plane [5].

\section{Formulation OF CELlS}

We now examine how we form cells on the earth's surface using an AP. Consider first the formulation of a single cell by a beam from a multibeam antenna system connected to the underside of the AP. The beam at an angle $\left(\theta_{i}, 0\right)$ from the AP delivers a footprint on the earth's surface, as shown in Fig. 9. This footprint corresponds to the half-power beamwidth, and we refer to this footprint as a single cell. Beyond this footprint, the coverage decreases with distance and constitutes interference in neighboring cells formed by other beams.

Referring to Fig. 9, the equation of the footprint for a beam with elevation plane half-power beamwidth $\mathrm{BW}_{\theta}=2 \theta_{h}$ and

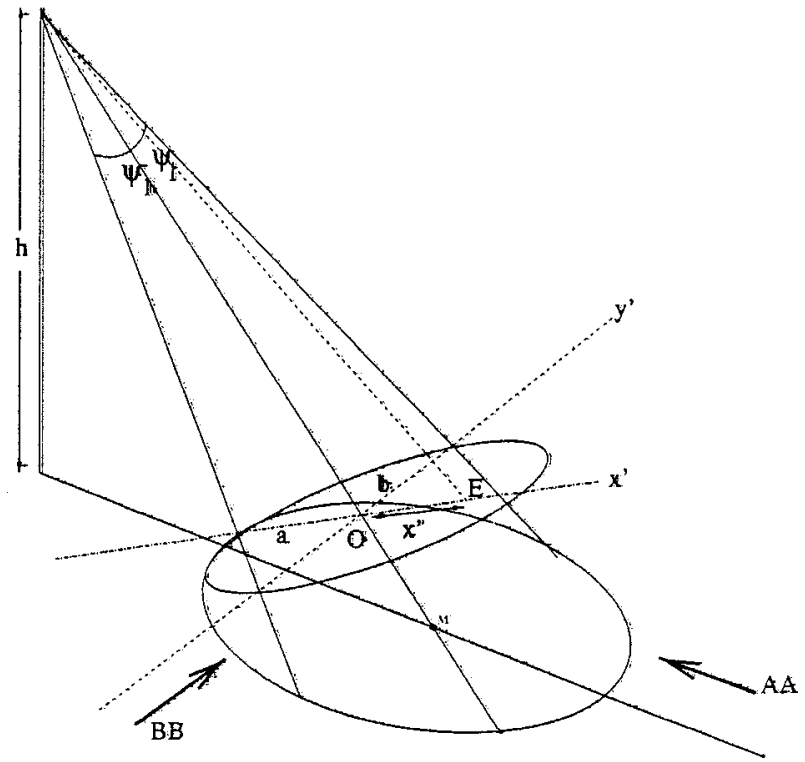

Fig. 9. The geometry of a footprint of a beam on the earth's surface from an AP.

half-power beamwidth $\mathrm{BW}_{\phi}=2 \phi_{h}$, in the plane that is perpendicular to the $\phi=\phi_{0}$ elevation, is shown in Appendix 1 to be

$$
\begin{aligned}
& y= \pm h \tan \left(\phi_{h}\right) \cos \left(\theta_{i}\right)\left(1+\frac{x}{h} \tan \left(\theta_{i}\right)\right) \\
& \cdot \sqrt{1-\left(\frac{1}{\tan \left(\theta_{h}\right)}\right)^{2}\left\{\frac{\frac{x}{h}-\tan \left(\theta_{i}\right)}{1+\frac{x}{h} \tan \left(\theta_{i}\right)}\right\}^{2}}
\end{aligned}
$$

where $h \tan \left(\theta_{i}-\theta_{h}\right) \leq x \leq h \tan \left(\theta_{i}+\theta_{h}\right)$. If a point $(x, y)$ is rotated by an angle $\phi_{i}$, the new coordinates, which are due to a beam directed toward $\left(\theta_{i}, \phi_{k}\right)$, becomes

$$
x^{\prime}=x \cos \left(\phi_{k}\right)-y \sin \left(\phi_{k}\right)
$$

and

$$
y^{\prime}=y \cos \left(\phi_{k}\right)+x \sin \left(\phi_{k}\right) .
$$

When $\theta_{i}=0$ and $\phi_{k}=0$, the equation is that of a circle of radius $h \tan \left(\theta_{h}\right)$, where $\theta_{h}$ is half of the half-power beamwidth of the broadside beam.

Let us consider an AP with a square array of $10 \times 10$ elements with $d=\lambda / 2$. As data from worldwide measurements of stratospheric wind velocities indicate that their minimum averages from 30 to $40 \mathrm{~m} / \mathrm{s}$ occur between 65000 and $75000 \mathrm{ft}$ (19-23 $\mathrm{km}$ ) depending on the latitude [7], we will consider the platform to be at a height of $21 \mathrm{~km}$ throughout this paper. The broadside half of the half-power beamwidth of this array is found numerically to be $5.1^{\circ}$, and the corresponding broadside cell radius is $1.876 \mathrm{~km}$. To find the footprints of different beams in different directions, we take into account the change of the beamwidth as the scanning angle changes by introducing a continuous function. To arrive at this continuous function, we first determined numerically the $\mathrm{HPBW}$ at $\theta_{i}=0^{\circ}, 10^{\circ}, 20^{\circ}, 30^{\circ}, 40^{\circ}$, and $50^{\circ}$ by setting (33) to 0.707 of its maximum value. The HPBW versus scanning angles for the above values of $\theta_{i}$ are plotted in Fig. 10 and titled "exact." The minimum least squares error 


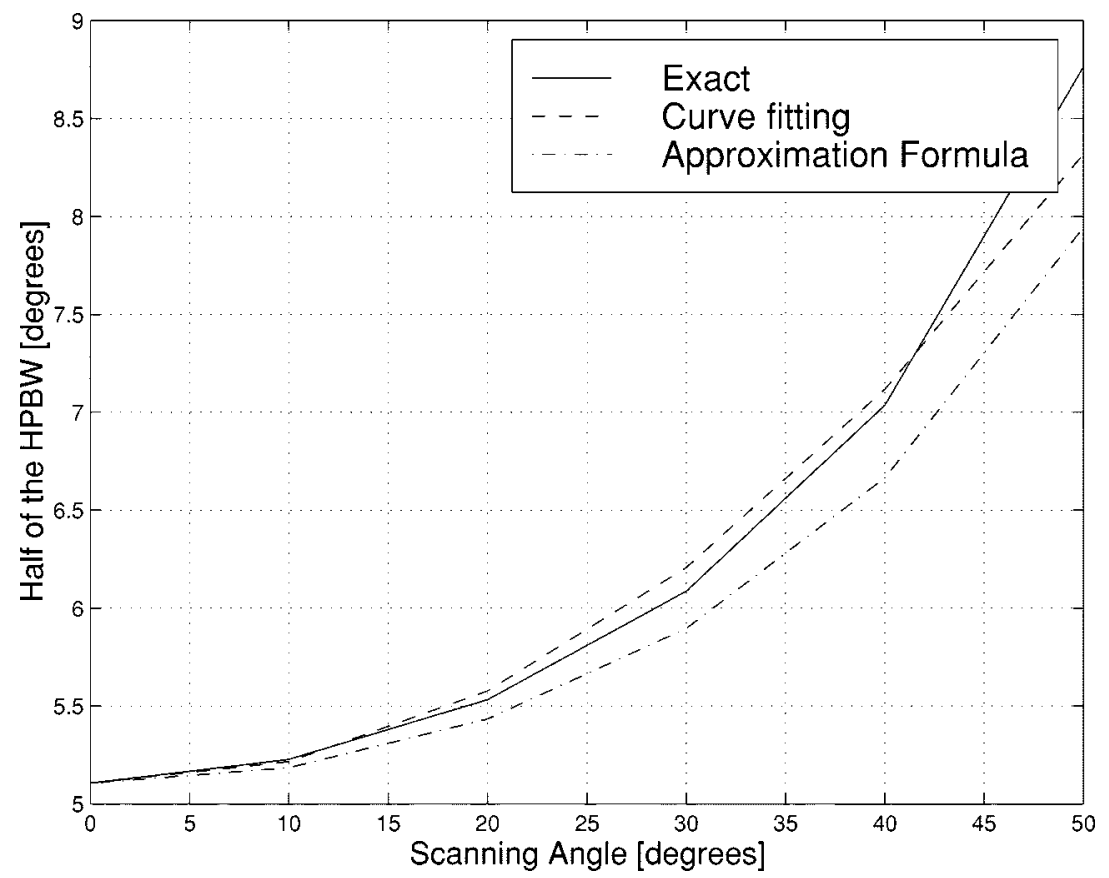

Fig. 10. The HPBW versus scanning angle for the exact results, using the approximation formula and the curve fitting procedure for a square array of $10 \times 10$ elements with spacing $d=\lambda / 2$.

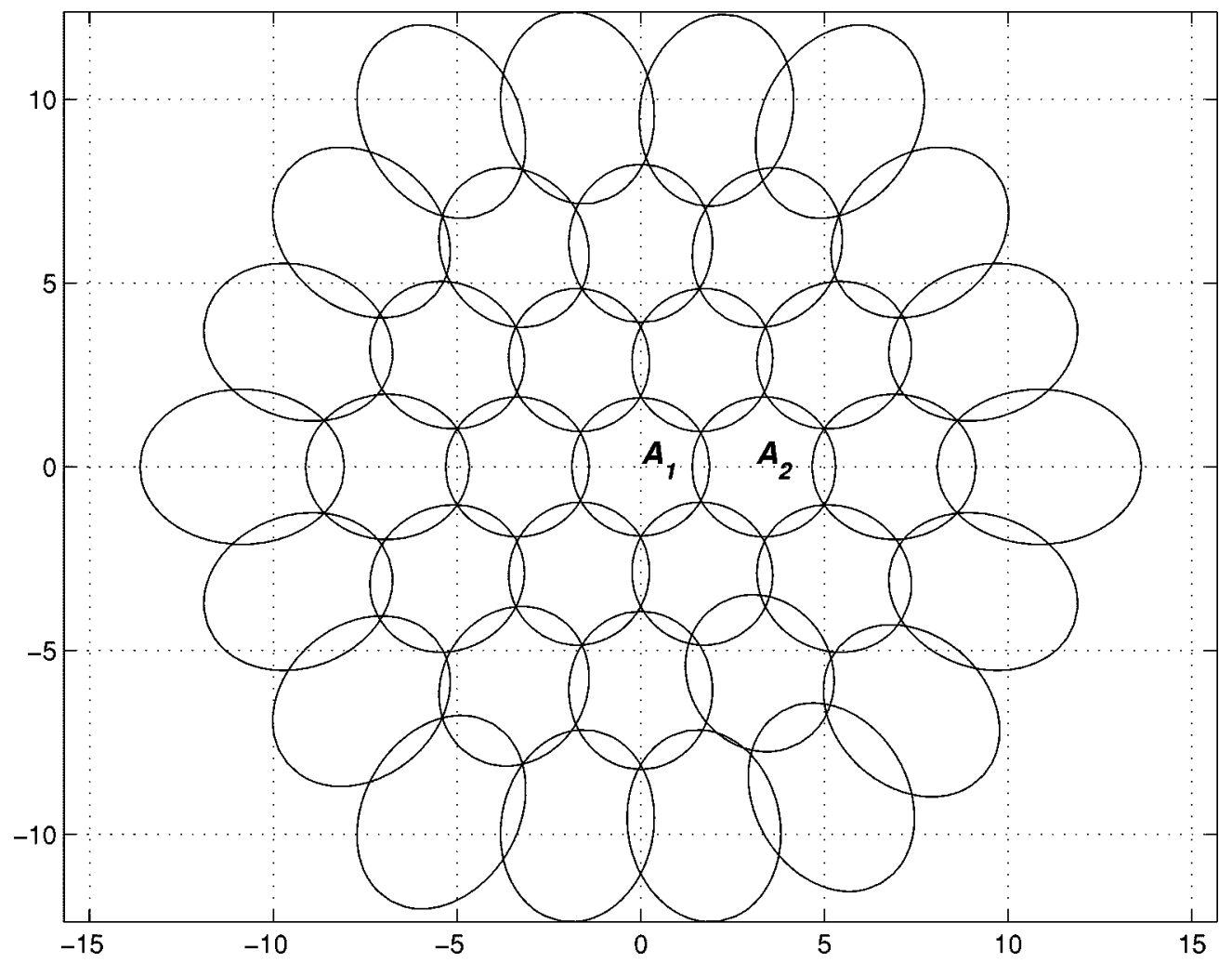

Fig. 11. Cell structure on the earth's surface for a square array having $10 \times 10$ elements with spacing $d=\lambda / 2$ (dimensions of both axes in km).

curve fitting was applied to the exact curve at the $\theta_{i}$ values to yield the continuous function of the half of the HPBW $\theta_{h}$

$$
\theta_{h}=0.0735698 \theta_{i}^{2.0866}+0.08911
$$

where $0 \leq \theta_{i} \leq 50^{\circ}$ (but converted to radians), such that $\theta_{i}$ is the direction of the main beam in the $\theta$-direction. This function is plotted in Fig. 10 and labeled "curve fitting." Also displayed in Fig. 10 is the curve "approximation formula" resulting from the application of (35). Notice that this curve has greater inaccuracy at higher scanning angles. The cell structure obtained from this array using (38) with the help of the curve fitting of (41) is shown in Fig. 11; see [4]. 


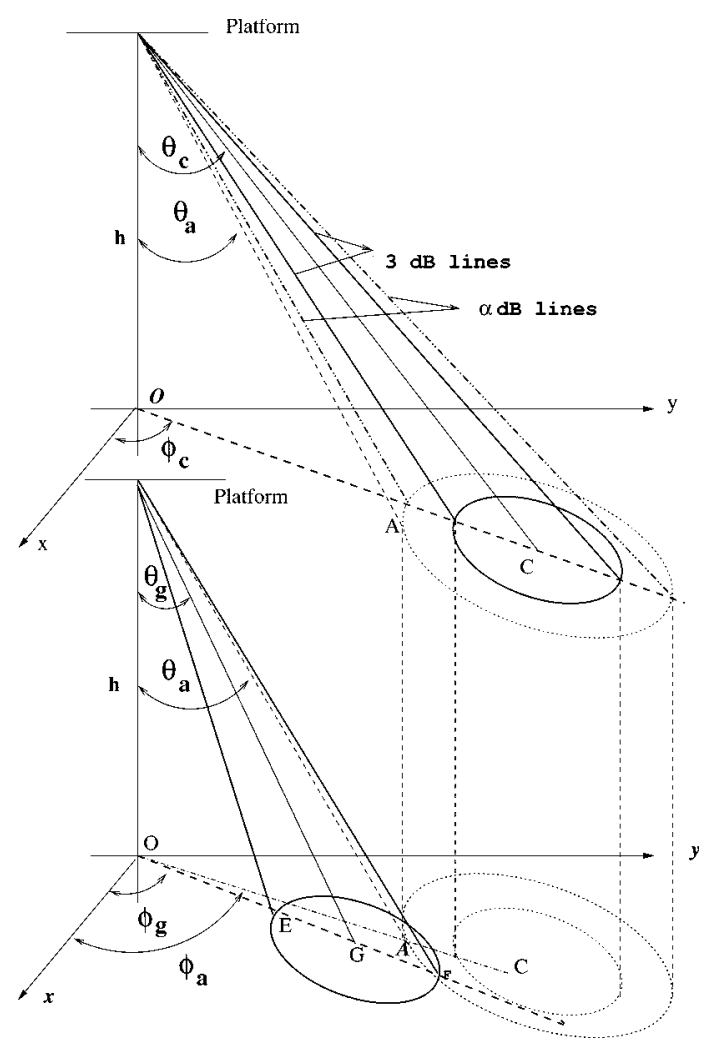

(a)

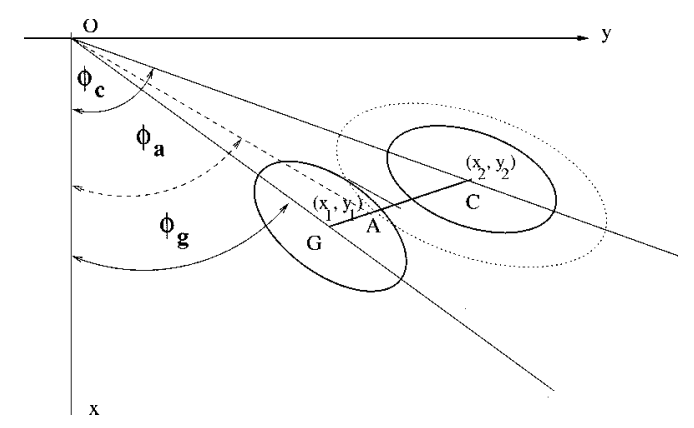

(b)

Fig. 12. Geometries employed in cochannel interference calculations.

\section{Contiguous Cells}

An AP at a height of $h \mathrm{~km}$ transmits and receives contiguous spot beams that form cells on the earth's surface. Each cell is defined here as an area within the HPBW coverage. Consider a mobile station (MS) located at point A in Fig. 12, which is $d_{a} \mathrm{~km}$ from the platform with direction $\left(\theta_{a}, \phi_{a}\right)$ measured from the position of the platform. This point is covered by a beam directed toward point $\mathrm{G}$ at direction $\left(\theta_{g}, \phi_{g}\right)$.

Let the power transmitted by the AP in each beam (i.e.,to each cell) be $P_{0}$. On assuming free-space propagation, the power received by a terminal at point $\mathrm{A}$ is

$$
P_{R}=P_{0} d_{a}^{-2} G_{T}\left(\theta_{a}, \phi_{a}\right) G_{R}
$$

where $G_{R}$ is the gain of the receiving antenna of the terminal and $G_{T}\left(\theta_{a}, \phi_{a}\right)$ is the gain of the beam covering the cell containing point A evaluated in the direction $\left(\theta_{a}, \phi_{a}\right)$. As the HPBW defines the cells, some energy is radiated into neighboring cells. The interference received by the MS from the main beam covering the cell centered at $\mathrm{C}$ is given by

$$
P_{I}=P_{0} d_{a}^{-2} G_{I}\left(\theta_{a}, \phi_{a}\right) G_{R}
$$

where the subscript $I$ signifies interference and $G_{I}\left(\theta_{a}, \phi_{a}\right)$ is the gain of the beam directed toward point $\mathrm{C}$ evaluated at $\left(\theta_{a}, \phi_{a}\right)$. The signal-to-interference ratio (SIR) at the MS is found from (42) and (43) as

$$
\mathrm{SIR}=\frac{P_{R}}{P_{I}}=\frac{G_{T}\left(\theta_{a}, \phi_{a}\right)}{G_{I}\left(\theta_{a}, \phi_{a}\right)} .
$$

For $J$ interfering cells, the total SIR is given by

$$
\mathrm{SIR}_{\text {Total }}=\frac{G_{T}\left(\theta_{a}, \phi_{a}\right)}{\sum_{j=1}^{J} G_{I j}\left(\theta_{a}, \phi_{a}\right)}
$$

where $G_{I j}\left(\theta_{a}, \phi_{a}\right)$ is the beam gain of the $j$ th interfering cell evaluated at $\left(\theta_{a}, \phi_{a}\right)$. For each service, the SIR should exceed a minimum value $\mathrm{SIR}_{\min }$. To fulfill this obligation, the following inequality should be satisfied:

$$
\sum_{j=1}^{J} G_{I j}\left(\theta_{a}, \phi_{a}\right) \leq \frac{G_{T}\left(\theta_{a}, \phi_{a}\right)}{\left(\operatorname{SIR}_{\min }\right)}
$$

The SIR of (45) can be calculated along a line connecting the centers of any two cochannel cells. Then the cell structure that satisfies the conditions in (46) can be determined. Assuming that the coordinate of point $\mathrm{G}$ in Fig. 12 is $\left(x_{1}, y_{1}\right)$, and that of point $\mathrm{C}$ is $\left(x_{2}, y_{2}\right)$, then the equation of line $\mathrm{GC}$ is

$$
y=m\left(x-x_{1}\right)+y_{1}
$$

where $x_{1} \leq x \leq x_{2}$ such that

$$
m=\frac{y_{2}-y_{1}}{x_{2}-x_{1}}
$$

from which the other distances and angles can be found as

$$
\begin{aligned}
\mathrm{GA} & =\sqrt{\left(y-y_{1}\right)^{2}+\left(x-x_{1}\right)^{2}} \\
\phi_{a} & =\tan ^{-1}\left(\frac{y}{x}\right) \\
\mathrm{OA} & =\sqrt{y^{2}+x^{2}} \\
\theta_{a} & =\tan ^{-1}\left(\frac{\mathrm{OA}}{h}\right) .
\end{aligned}
$$

The SIR and the required cell structure will be calculated for an $\mathrm{AP}$ at a height of $21 \mathrm{~km}$ employing a square array of $10 \times 10$ elements such that the distance between each two elements is $d=\lambda / 2$.

1) Single Cell Per Cluster: Assume that the AP is used for the UMTS system, for which some of the characteristics are shown in Table I. 
TABLE I

SOME UMTS SPECIFICATIONS [8]

\begin{tabular}{c||l|l}
\hline S. No. & Item & Description \\
\hline 1 & Frequency Band & $1.88 \mathrm{GHz}-2.2 \mathrm{GHz}$ \\
\hline 2 & Channel BW & $4.4 \mathrm{MHz}$ \\
\hline 3 & Service bit rate & $0-2048 \mathrm{~Kb} / \mathrm{s}$ \\
\hline 4 & $\begin{array}{l}\text { RF Channel } \\
\text { Chip Rate }\end{array}$ & $\begin{array}{l}4.096,8.192, \\
16.384 \mathrm{Mcps}\end{array}$ \\
\hline 5 & $\begin{array}{l}\text { Maximum power } \\
\text { transmitted }\end{array}$ & $30 \mathrm{dBm}$ \\
\hline 6 & $\begin{array}{l}\text { Total Delay } \\
\text { (round trip) }\end{array}$ & $25 \mathrm{~ms}$ \\
\hline 7 & $\begin{array}{l}\text { Base station } \\
\text { Noise Figure }\end{array}$ & $5 \mathrm{~dB}$ \\
\hline 8 & $\begin{array}{l}\text { Mobile station } \\
\text { Noise Figure }\end{array}$ & $5 \mathrm{~dB}$ \\
\hline 9 & $\begin{array}{l}\text { Cable, connector } \\
\text { and other losses }\end{array}$ & $2 \mathrm{~dB}$ \\
\hline 10 & $\begin{array}{l}\text { Required } \frac{E_{b}}{N_{0}+I_{0}} \\
\text { (Down link) }\end{array}$ & $0.2-7.9 \mathrm{~dB}$ \\
\hline
\end{tabular}

Arranging for all the beams to have the same maximum gain, i.e., $C_{i k}=C$ for all $i$ and $k$

$$
\begin{aligned}
G_{T}\left(\theta_{a}, \phi_{a}\right)=C^{2} \\
\cdot\left|\frac{\sin \left[\frac{\pi N}{2}\left\{\sin \left(\theta_{a}\right) \cos \left(\phi_{a}\right)-\sin \left(\theta_{g}\right) \cos \left(\phi_{g}\right)\right\}\right]}{\sin \left[\frac{\pi}{2}\left\{\sin \left(\theta_{a}\right) \cos \left(\phi_{a}\right)-\sin \left(\theta_{g}\right) \cos \left(\phi_{g}\right)\right\}\right]}\right|^{2} \\
\cdot\left|\frac{\sin \left[\frac{\pi N}{2}\left\{\sin \left(\theta_{a}\right) \sin \left(\phi_{a}\right)-\sin \left(\theta_{g}\right) \sin \left(\phi_{g}\right)\right\}\right]}{\sin \left[\frac{\pi}{2}\left\{\sin \left(\theta_{a}\right) \sin \left(\phi_{a}\right)-\sin \left(\theta_{g}\right) \sin \left(\phi_{i}\right)\right\}\right]}\right|^{2}
\end{aligned}
$$

and

$$
\begin{aligned}
G_{I}\left(\theta_{a}, \phi_{a}\right)=C^{2} \\
.\left|\frac{\sin \left[\frac{\pi N}{2}\left\{\sin \left(\theta_{a}\right) \cos \left(\phi_{a}\right)-\sin \left(\theta_{c}\right) \cos \left(\phi_{c}\right)\right\}\right]}{\sin \left[\frac{\pi}{2}\left\{\sin \left(\theta_{a}\right) \cos \left(\phi_{a}\right)-\sin \left(\theta_{c}\right) \cos \left(\phi_{c}\right)\right\}\right]}\right|^{2} \\
\cdot\left|\frac{\sin \left[\frac{\pi N}{2}\left\{\sin \left(\theta_{a}\right) \sin \left(\phi_{a}\right)-\sin \left(\theta_{c}\right) \sin \left(\phi_{c}\right)\right\}\right]}{\sin \left[\frac{\pi}{2}\left\{\sin \left(\theta_{a}\right) \sin \left(\phi_{a}\right)-\sin \left(\theta_{c}\right) \sin \left(\phi_{c}\right)\right\}\right]}\right|^{2}
\end{aligned}
$$

which is the gain of the beam of the interfering cell directed toward $\mathrm{C}$ evaluated at $\left(\theta_{a}, \phi_{a}\right)$. By selecting $C=0.316 \mathrm{~A}$, the maximum gain of the main beam is $30 \mathrm{~dB}$ as the array dimensions are 10 by 10 . Arranging for one cell per cluster, the gain of the main beam $A_{1}$ in Fig. 11 as a function of the distance from the center of $A_{1}$ along a line to the center of $A_{2}$ (also in Fig. 11) is shown by the solid line in Fig. 13(a). We emphasise that the the same frequencies are used in cells $A_{1}$ and $A_{2}$. Also displayed in Fig. 13(a) is the curve representing the variation of the gain of the main beam for $A_{2}$. Defining a cell as the 3-dB beamwidth, we note that the boundary of $A_{1}$ is at about $1.9 \mathrm{~km}$ (similar to the previous calculations), while the boundary of $A_{2}$ commences at about $1.4 \mathrm{~km}$.

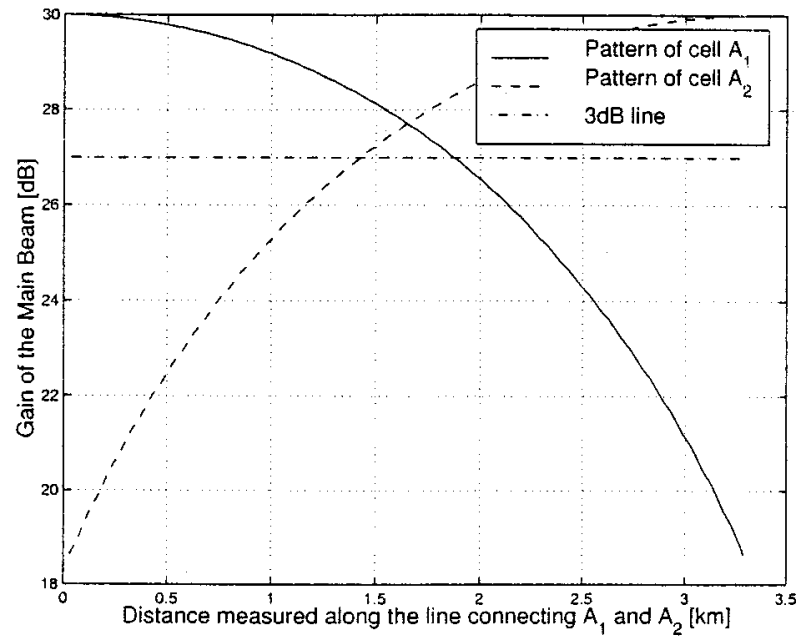

(a)

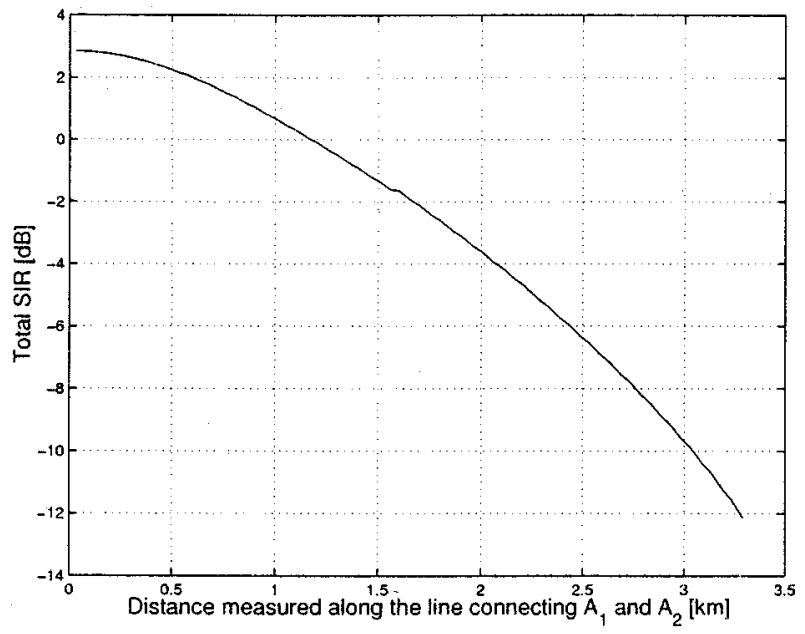

(b)

Fig. 13. Results for one cell per cluster.

Fig. 13(b) shows the variation of SIR experienced by an MS assigned to $A_{1}$ as it travels the same distance in Fig. 13(a). The interference is computed not just from cell $A_{2}$ but from all the cells that form a ring around $A_{1}$, assuming one user per cell. Observe that at the cell boundary of $A_{1}$, the SIR is about -3.5 $\mathrm{dB}$, and hence this cell cluster arrangement is only suitable for CDMA, and not time- or frequency-division multiple access.

In digital systems, the SIR at the receiver input is closely related to $E_{b} / I_{0}$ at baseband, namely [9]

$$
\operatorname{SIR}=\left(\frac{E_{b}}{I_{0}}\right)\left(\frac{R_{b}}{B_{c}}\right)
$$

where

$E_{b} \quad$ energy per bit;

$I_{0}$ interference power spectral density (PSD) in watts/ Hertz;

$R_{b} \quad$ message data rate in bits per second;

$B_{c} \quad$ radio channel bandwidth in hertz where $B_{c} \gg R_{b}$.

In CDMA, all users share one radio channel, each having a different code. If a CDMA system is deployed and there are $M$ active users per cell uniformly distributed over the cell, then each 
user contributes the same amount of interference such that the total SIR becomes

$$
\operatorname{SIR}=\frac{P_{0} G_{T}\left(\theta_{a}, \phi_{a}\right)}{P_{0}(M-1) G_{T}\left(\theta_{a}, \phi_{a}\right)+M P_{0} \sum_{j=1}^{J} G_{I j}\left(\theta_{a}, \phi_{a}\right)}
$$

or

$$
\operatorname{SIR}=\frac{1}{M(1+\gamma)-1}
$$

where

$$
\gamma=\frac{\sum_{j=1}^{J} G_{I j}\left(\theta_{a}, \phi_{a}\right)}{G_{T}\left(\theta_{a}, \phi_{a}\right)}
$$

is the inverse of the total SIR experienced by the mobile in the cell under considerations from the cochannel cells, assuming one mobile per cochannel cell [it is the inverse of (45)]. The value of $\gamma$ can be obtained with the help of Fig. 13(b).

Equating (55) and (57) and solving for $M$ gives

$$
M=\frac{\left(\frac{E_{b}}{I_{0}}\right)\left(\frac{R_{b}}{B_{c}}\right)+1}{(1+\gamma)\left(\frac{E_{b}}{I_{0}}\right)\left(\frac{R_{b}}{B_{c}}\right)}
$$

where we observe that the number of users decreases as $\gamma$ increases. The worst case is on the cell boundary, when $M$ represents the lower bound of the number of users the system can accommodate, assuming the other variables of (5) are constants. Consider the central cell, from Fig. 13(b), $\gamma=3.5 \mathrm{~dB}$ on the boundary of the central cell. Using this value for $\gamma$ and for $B_{c}=4.4 \mathrm{MHz},(59)$ was calculated as a function of $E_{b} / I_{0}$ for different service rates. The results are displayed in Fig. 14(a). The same equation was calculated as a function of the service rate for different values of $E_{b} / I_{0}$, and the results are displayed in Fig. 14(b). From Fig. 14(a), we observe that the system can accommodate more than 160 users having a service rate of $8 \mathrm{~kb} / \mathrm{s}$ if the required $E_{b} / I_{0}$ is $0.2 \mathrm{~dB}$, and 30 users if $E_{b} / I_{0}=7.9 \mathrm{~dB}$. These numbers drops to 40 and eight users, respectively, if the service rate is increased to $32 \mathrm{~kb} / \mathrm{s}$. From Fig. 14(b), the number of users decreases sharply as the service rate increases above 32 $\mathrm{kb} / \mathrm{s}$.

2) Three Cells Per Cluster: When the procedure is applied for the three cells per cluster arrangement shown in Fig. 15, we obtain the variation of the gain of the main beam $B_{1}$, and the main beam toward $B_{2}$, as a function of distance measured along the line connecting the two cells. The result is shown in Fig. 16(a). From Fig. 15, we calculate the interference experienced by the central cell $B_{1}$ from cells $B_{-3}, B_{-2}, B_{-1}, B_{2}, B_{3}$, and $B_{4}$. The SIR is found next, and its variation with distance is shown in Fig. 16(b). From Fig. 16(a), the boundary of the central cell $B_{1}$ is also at approximately $1.9 \mathrm{~km}$, while the boundary of the cell $B_{2}$ is at about $4 \mathrm{~km}$. From Fig. 16(b), the corresponding distance to $\mathrm{SIR}=8 \mathrm{~dB}$ is $2 \mathrm{~km}$. As this distance is between

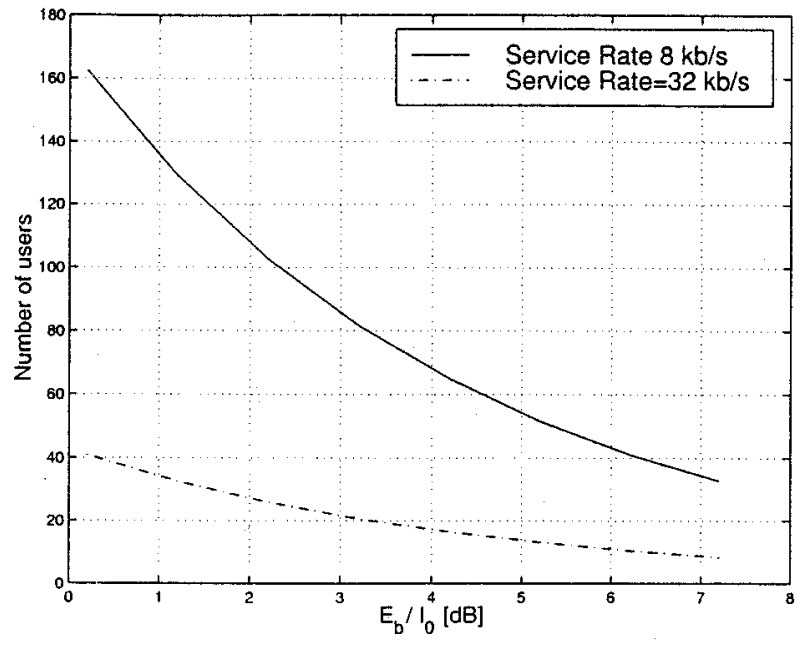

(a)

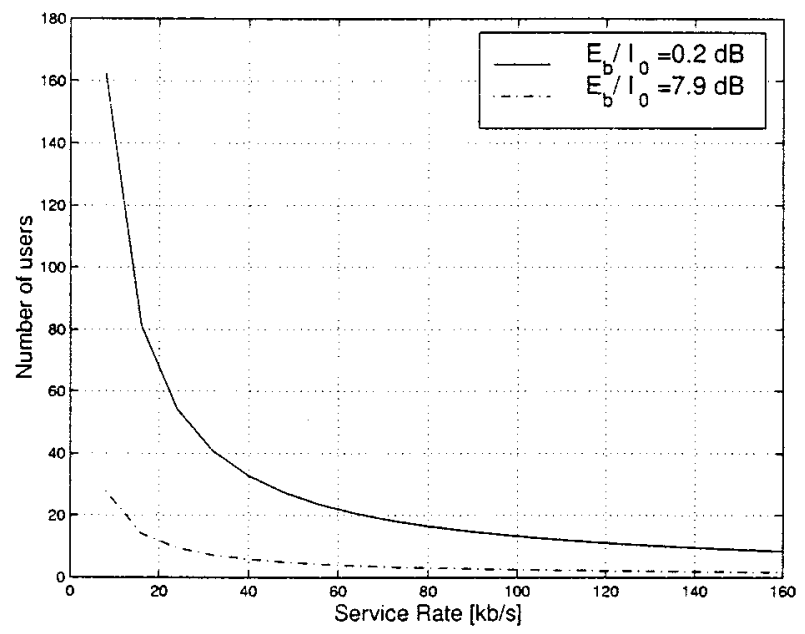

(b)

Fig. 14. Minimum number of users with UMTS CDMA.

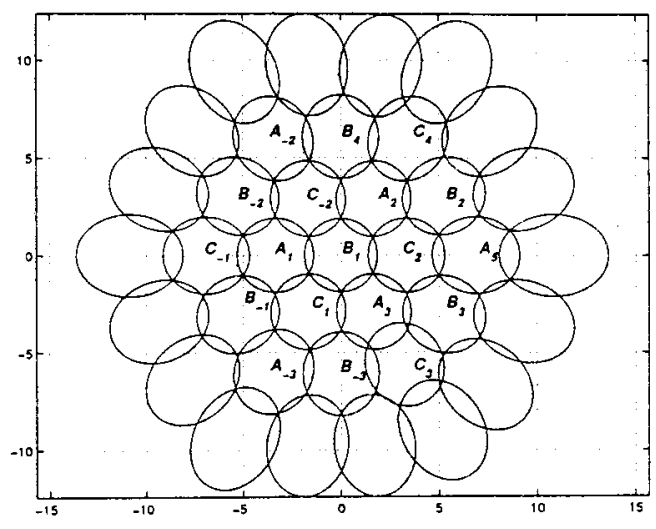

Fig. 15. Cell structure for square array of $10 \times 10$ elements with spacing $d=$ $\lambda / 2$ with a reuse factor of three. Dimensions are in kilometers.

the boundaries of $B_{1}$ and $B_{2}$, we conclude that three cells per cluster are also suitable for third-generation TDMA systems.

\section{Cells with Variable Sizes and Locations}

The cells formed by the AP can be varied in both their size and location. The arrangement to control these changes is shown 


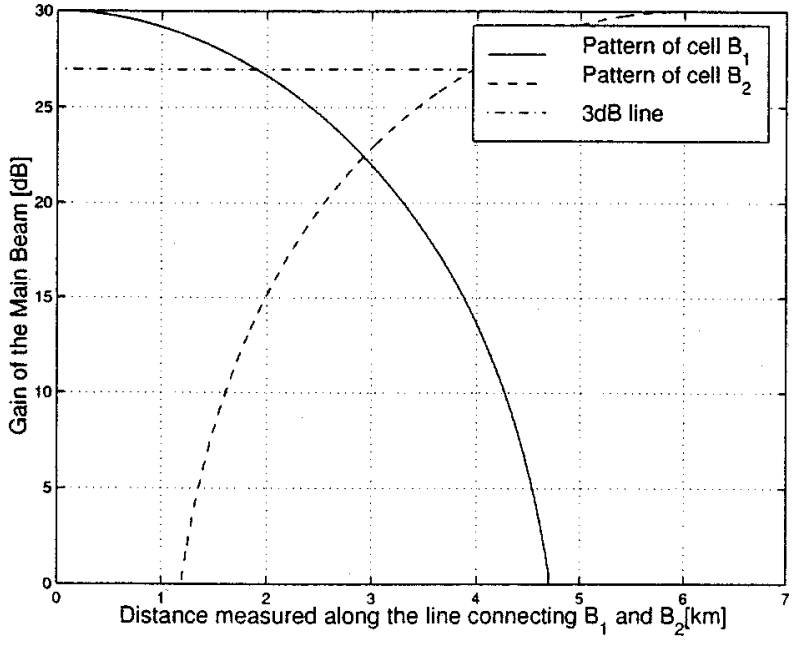

(a)

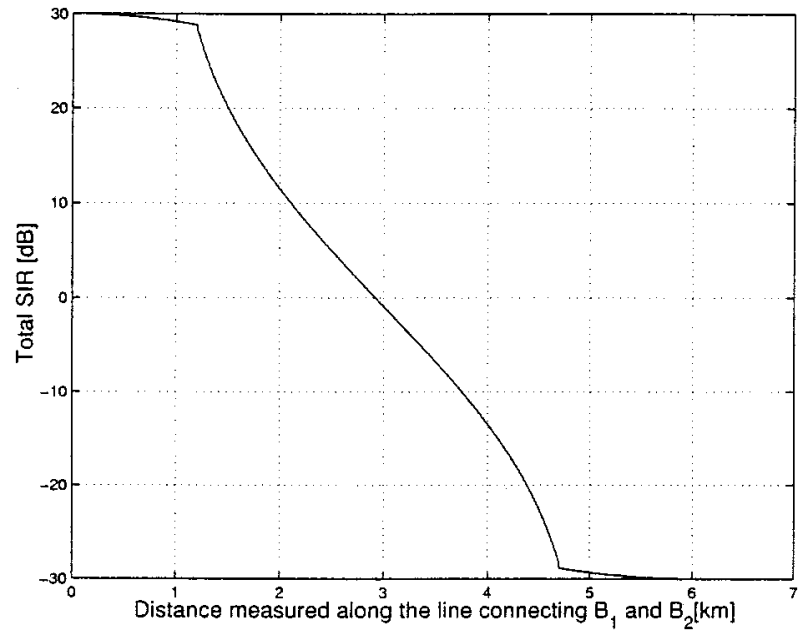

(b)

Fig. 16. Results for three cells per cluster.

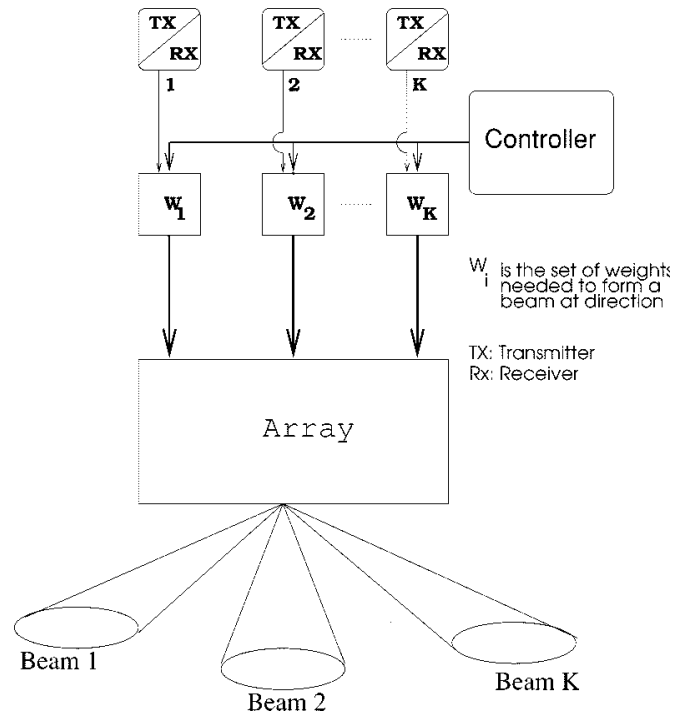

Fig. 17. Arrangements to change cell size and location.

in Fig. 17. The controller changes the phase of the weights according to the required direction, the number of weights to the

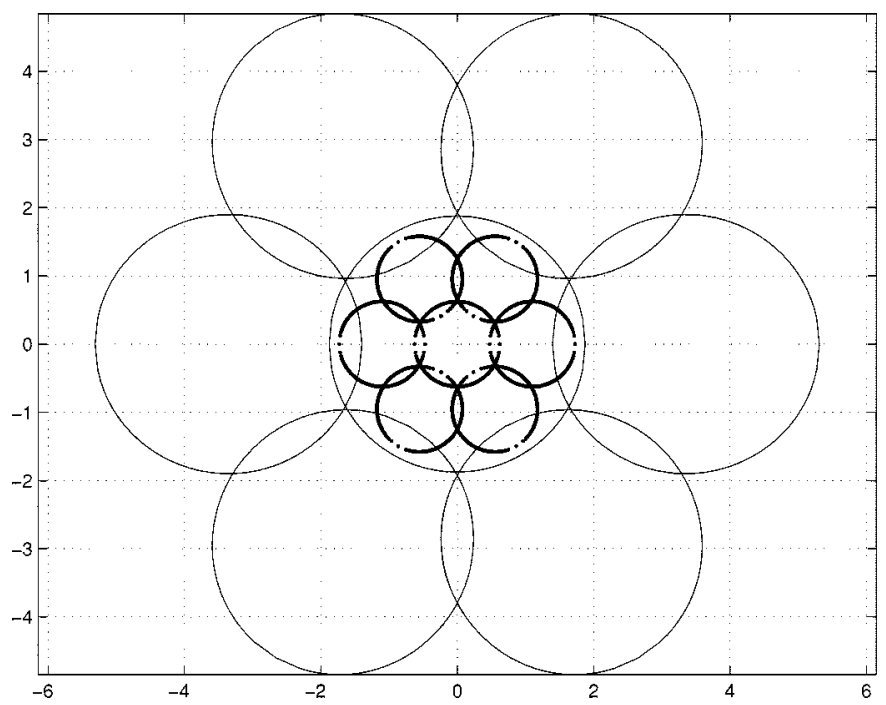

(a)

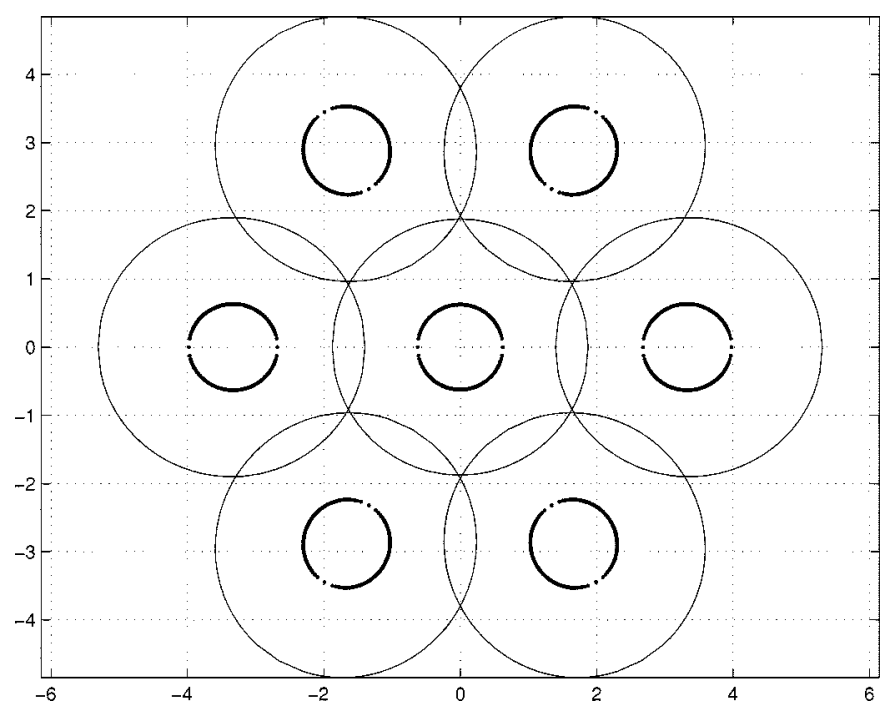

(b)

Fig. 18. Cell forming according to traffic.

size(number of connected elements of the array) according of the cell, and the values of the weight to adjust the gain of the beam.

As an example of the flexibility to form macrocells and microcells, we consider the arbitrary scenario depicted in Fig. 18. The microcells are realized using a square array of $30 \times 30$ elements, and the macrocells are established by an array $10 \times 10$ elements. We will probably need to use one CDMA carrier for the microcells and another for the macrocells. Let us suppose that the AP is above the city center and at noon we provide seven microcells and one macrocell over the business district, while suburbia has six macrocells. In the morning and early evening, mobiles are entering and leaving the business district, respectively. The microcells follow the offered traffic as it spatially and temporally changes. By late evening, the microcells can be positioned throughout the center as required. For purposes of example, we have placed them at the center of each macrocell in Fig. 18(b). The essential point is that variable cells can be formed and moved to accommodate spatially and time-varying traffic offered by the MSs. 


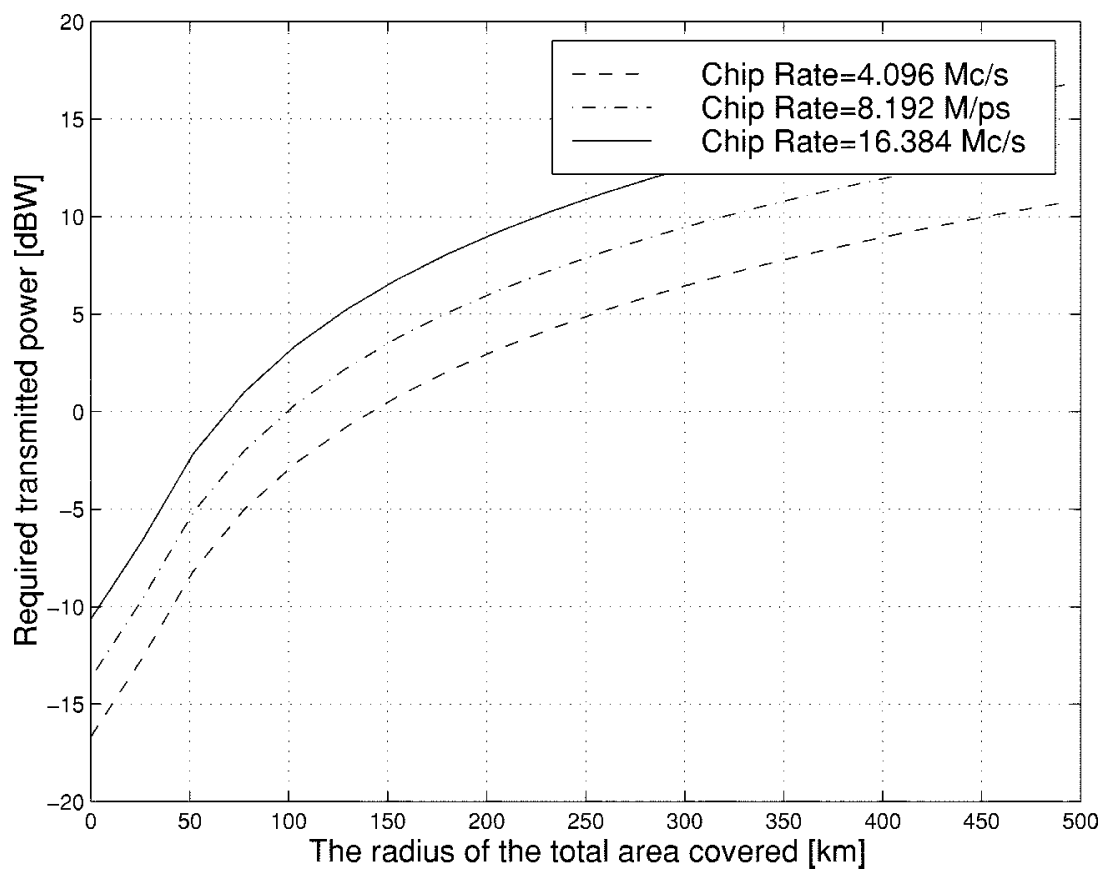

Fig. 19. The required transmitted power versus the radius of the area covered for different chip rates.

\section{TRANSMISSION RATE AND DELAY}

The maximum propagation time delay $\tau_{p}$ of a signal transmitted by the platform can be calculated as

$$
\tau_{p}=\frac{2 L}{c}
$$

where $c$ is the speed of light $\left(=3 \times 10^{8} \mathrm{~m} / \mathrm{s}\right)$ and $L$ is the distance shown in Fig. 1 and given by (2).

Delay is an important factor in global communications. It is interesting to compare the delay of an AP network with the delay incurred in a fiber link. To make this comparison, we consider the arc length $\mathrm{AB}$ in Fig. 1, as it is the maximum distance between two mobiles. The distance using optical transmission is the length of the arc AB. Applying (7) for $L / R_{e}<8 \pi / 180$, $\tan \left(L / R_{e}\right) \cong\left(L / R_{e}\right)$, yielding the $\operatorname{arc} A B=2 L$ for $L<900$ $\mathrm{km}$. For the AP, the maximum distance travelled by a signal is $2 \times 517 \mathrm{~km}$ for a platform at $21 \mathrm{~km}$. The optical signal travels almost the same distance. However, the propagation of light in fiber depends on its dielectric constant. If it is $2 \times 10^{8} \mathrm{~m} / \mathrm{s} \mathrm{com}$ pared to $3 \times 10^{8} \mathrm{~m} / \mathrm{s}$ for the velocity of light, the time taken to travel arc AB for the optical fiber link and for the AP links is 5.2 and $3.5 \mathrm{~ms}$, respectively.

When transactions are relatively local, then delay constraints can be relaxed, but attention must be given to the minimization of delay when we are considering transactions on a global basis. As an example, for an ocean of $6000 \mathrm{~km}$, a geostationary link is $250 \mathrm{~ms}$, a fiber of length $10000 \mathrm{~km}$ (to allow for variations on the ocean floor) is $50 \mathrm{~ms}$, while if we establish a network of sky platforms [3] at $20 \mathrm{~km}$ above the ocean, spaced $1 \mathrm{~km}$ apart, the delay is only $23 \mathrm{~ms}$, ignoring the relaying process delay in each sky platform [10]. Low-earth-orbit satellites have delays only a little greater than those of sky platform transmissions, but they have disadvantages of a relatively low capacity and high Doppler frequency shifts [10].
For a digital free-space channel, the bit energy per noise power spectral density $E_{b} / N_{0}$ is given by [11]

$$
\frac{E_{b}}{N_{o}}=\frac{P_{t} G_{t} G_{r}}{R_{b} K T^{\circ} L_{s} L_{o} M_{L}}
$$

where

$R_{b} \quad$ transmission rate in bits per second;

$T^{\circ}$ receiver temperature in degrees Kelvin;

$K \quad$ Boltzmann's constant $\left(=1.38 \times 10^{-23} \mathrm{~J} / \mathrm{K}\right)$;

$L_{s} \quad$ free-space loss;

$L_{0} \quad$ summation of other losses (e.g circuits losses);

$M_{L}$ link margin.

The free-space loss is expressed as [11]

$$
L_{s}=\left(\frac{4 \pi d_{L}}{\lambda}\right)^{2}
$$

where $d_{L}$ is the link distance and $\lambda$ is the wavelength. Expressed in decibels, (61) becomes

$$
\begin{aligned}
\frac{E_{b}}{N_{o}}(\mathrm{~dB})= & P_{t}(\mathrm{~dB})+G_{t}(d B \mathrm{~dB})+G_{r}(\mathrm{~dB}) \\
& -10 \log \left(R_{b}\right)-10 \log \left(K T^{\circ}\right) \\
& -10 \log \left(L_{s}\right)-\log \left(L_{0}\right)-10 \log \left(M_{L}\right) .
\end{aligned}
$$

and the free-space loss (62) becomes

$$
L_{s}=20 \log \left(f_{0}\right)+20 \log \left(d_{L}\right)-147.56
$$

where $f_{0}$ is the operating frequency in hertz and $d_{L}$ is the link distance in meters. The link distance depends on the location of the station measured from the AP and is calculated using (11).

The required transmitted power of the AP at a height of 21 $\mathrm{km}$ is calculated for the chip rates of 4.096, 8.192, and 16.384 $\mathrm{Mc} / \mathrm{s}$. In these calculations, the operating frequency used is 2.2 $\mathrm{GHz}$, the link margin is $15.4 \mathrm{~dB}$, the required $E_{b} / N_{0}=7.9$, and the loss $L_{0}$ due to cable, connector, and combiner losses is $2 \mathrm{~dB}$. The calculation assumed $30 \mathrm{dBi}$ for the antenna on the AP and 


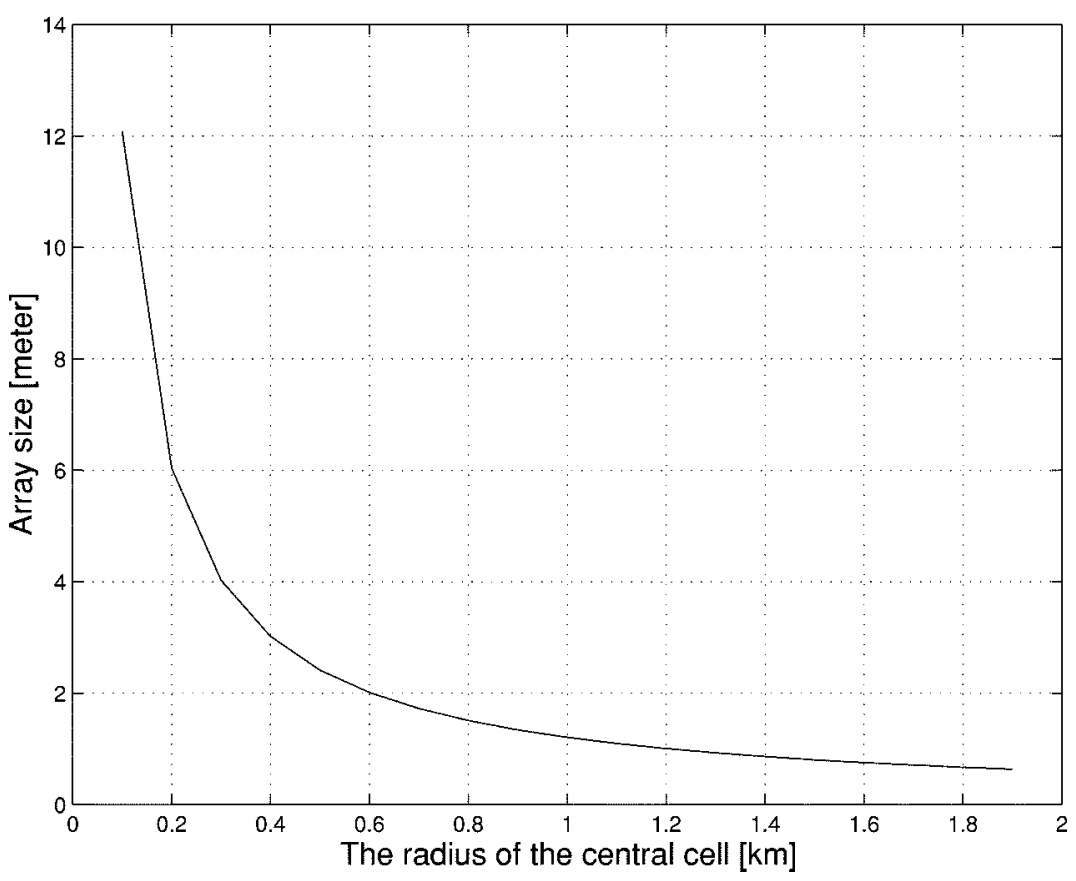

Fig. 20. Array size as a function the radius of the central cell at $21 \mathrm{~km}$ and $2.2 \mathrm{GHz}$.

an isotropic antenna for mobile station. The transmitted power as a function of the radius of the total area covered is displayed in Fig. 19. Observe that an AP at a height of $21 \mathrm{~km}$ can cover an area of radius $70 \mathrm{~km}$ with a chip rate of up to $16.384 \mathrm{Mc} / \mathrm{s}$ using $0 \mathrm{~dB}(1 \mathrm{~mW})$ transmission power.

\section{ARRAY Structure SizE}

As an adaptive antenna array system will be used on the AP, the structure size is of importance. For large square arrays, the broadside $3-\mathrm{dB}$ beamwidth (HPBW) is approximated by [6]

$$
B W_{\theta}=\frac{2.65 \lambda}{\pi N d} .
$$

The radius $r_{c}$ of the broadside cell (the central cell) formed by an AP at height $h$ is

$$
r_{c}=h \tan \left(\theta_{h}\right)=h \tan \left(\frac{0.42176 \lambda}{L_{A}}\right)
$$

where $\theta_{h}=\frac{1}{2} \mathrm{BW}_{\theta}$ and $L_{A}=N d$ is the size of the array. Substituting for $\lambda=c / f$, the size of the array may be calculated using

$$
L_{A}=0.42176 \frac{c}{f_{0} \tan ^{-1}\left(\frac{r_{c}}{h}\right)} .
$$

For a specified $r_{c}$, increasing the height of the AP requires a larger array. However, the array size decreases as the operating frequency increases. The array size as a function of the radius of the central cell for $h=21 \mathrm{~km}$, assuming operating frequency of $2.2 \mathrm{GHz}$, is shown in Fig. 20. From this figure, it is clear that the size of the arrays needed in the AP system is realizable.

\section{CONCLUSION}

With the expected deployment of third-generation (3G) networks starting in 2002, the application of aerial platforms in $3 \mathrm{G}$ is very attractive. For our part, we have conducted a preliminary analysis that gives us optimism that aerial platforms have a future role to play in supporting $3 \mathrm{G}$ terrestrial multimedia networks. They have the ability to provide multiple cells via the adaptive multibeam antenna arrays they have on-board; to change the beam size to form cells as small as $100 \mathrm{~m}$, i.e., form microcells; and to generate large cells of many kilometers if required. The platforms are stationary so they do not introduce any changes in Doppler frequency, they are at a sufficiently low altitude that propagation delays are only a few milliseconds, and their capacity can be very high because they can operate CDMA links with a reuse of unity. The movement of the beams means the cells move, and if necessary, simultaneously change in size. Therefore, the infrastructure can be electronically reorganized to suit the changing teletraffic patterns of mobile users. Notice that the cost of terrestrial base stations and site rentals is now transformed to the cost of the aerial platforms.

We have examined the basic parameters of platform height, coverage, cell size, array size, transmitted power, and so forth, and found them satisfactory. More quantifying of the parameters is needed before the details of an aerial $3 \mathrm{G}$ system can be precisely formulated. In particular, the performance of the CDMA uplink needs quantifying, a more complex propagation law that takes into account the effects of terrain and buildings needs to be used, and the effect of antenna side-lobes on a cochannel interference must be examined, as well as perturbations on the position of the AP. Nevertheless, we trust that this paper will be valuable as a step along the path to the deployment of APs for 3G systems. 


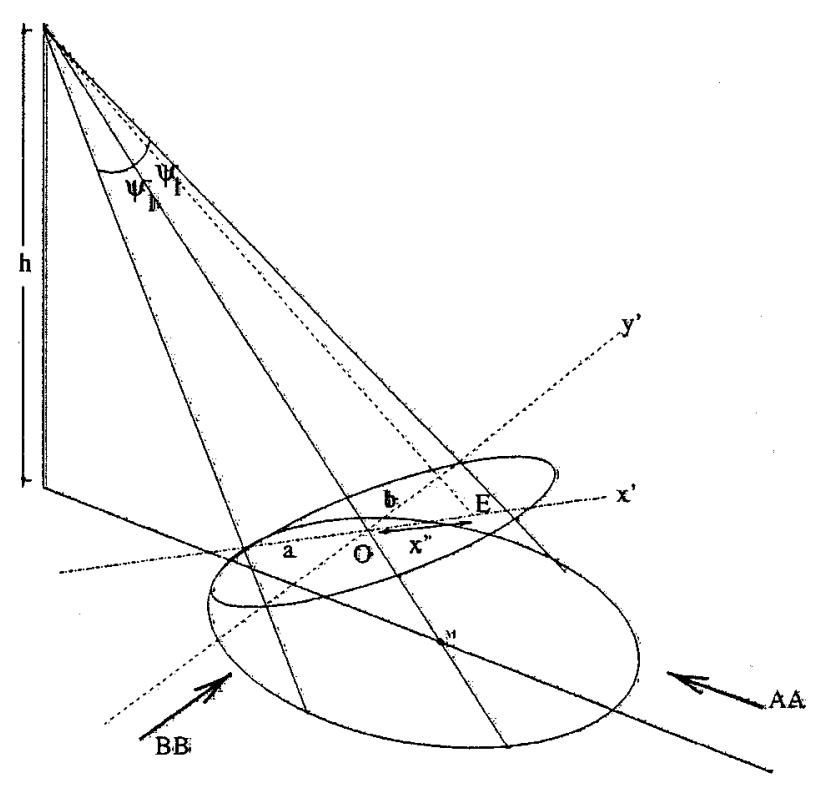

(a)

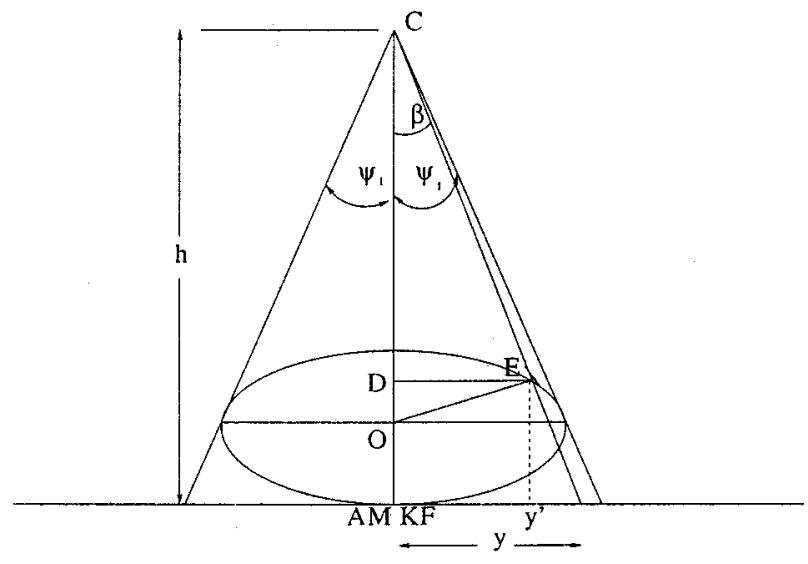

(b)

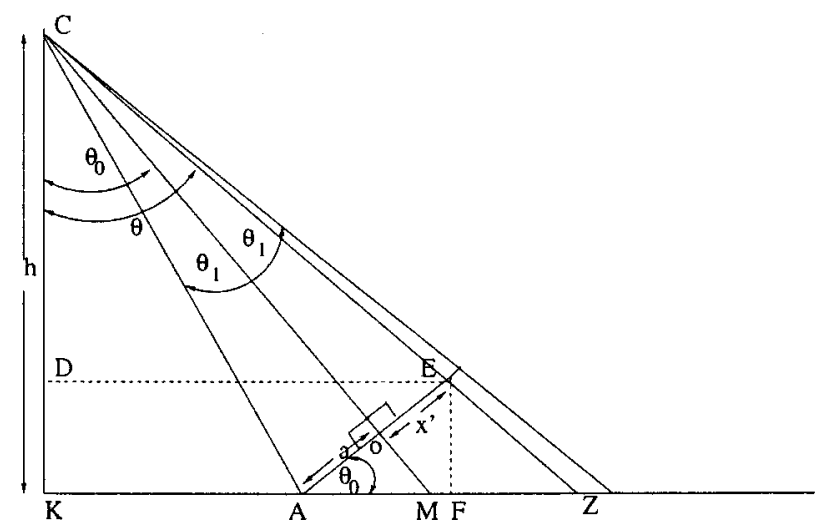

(c)

Fig. 21. Geometry for one beam from the AP.

APPENDIX A

FOOT-PRINT OF THE AP BEAMS

From Fig. 21(c), the distance $x=A Z$ is given by

$$
x=h\left[\tan (\theta)-\tan \left(\theta_{0}-\theta_{1}\right)\right]
$$

and

$$
x^{\prime}=\mathrm{OC} \tan \left(\theta-\theta_{0}\right)
$$

where $\theta_{0}-\theta_{1} \leq \theta \leq \theta_{0}+\theta_{1}$. Also

$$
y=h \tan (\beta) \text {. }
$$

The equation of the ellipse formed by intersection of the beam with the plane $x^{\prime}-y^{\prime}$ is

$$
\frac{x^{2}}{a^{2}}+\frac{y^{2}}{b^{2}}=1
$$

where

$$
a=\mathrm{OC} \tan \left(\theta_{1}\right)
$$

and

$$
b=\mathrm{OC} \tan \left(\psi_{1}\right)
$$

from which

$$
y^{\prime}= \pm b \sqrt{1-\frac{x^{\prime 2}}{a^{2}}}
$$

From the projection in Fig. 21(c)

$$
E F=\left(a+x^{\prime}\right) \sin \left(\theta_{0}\right) \text {. }
$$

The line OC $=\mathrm{CM}-\mathrm{MO}$, so

$$
O C=\frac{h}{\cos \left(\theta_{0}\right)}-a \tan \left(\theta_{0}\right) .
$$

Substituting (A.5) into (A.9) and solving for OC leads to

$$
\mathrm{OC}=\frac{h}{\cos \left(\theta_{0}\right)\left[1+\tan \left(\theta_{1}\right) \tan \left(\theta_{0}\right)\right]} .
$$

From Fig. 21(b)

$$
\tan (\beta)=\frac{y^{\prime}}{\mathrm{DC}}
$$

and

$$
\begin{aligned}
\mathrm{DC} & =h-\mathrm{EF} \\
& =h-\mathrm{OC}\left[\tan \left(\theta_{1}\right)+\tan \left(\theta-\theta_{0}\right)\right] \sin \left(\theta_{0}\right)
\end{aligned}
$$


Substituting (A.10) into (A.12) leads to

$$
\mathrm{DC}=h \frac{1-\tan \left(\theta_{0}\right) \tan \left(\theta-\theta_{0}\right)}{1+\tan \left(\theta_{1}\right) \tan \left(\theta_{0}\right)} .
$$

Substitute this result into (A.11); then substituting in (A.3) using (A.2) and (A.7) gives

$$
y= \pm \frac{h \tan \left(\psi_{1}\right) \sqrt{1-\left\{\frac{\tan \left(\theta-\theta_{0}\right)}{\tan \left(\theta_{1}\right)}\right\}^{2}}}{\cos \left(\theta_{0}\right)\left[1-\tan \left(\theta_{0}\right) \tan \left(\theta-\theta_{0}\right)\right]}
$$

Substituting the formula

$$
\tan \left(\theta_{0}-\theta\right)=\frac{\tan \left(\theta_{0}\right)-\tan (\theta)}{1+\tan (\theta) \tan \left(\theta_{0}\right)}
$$

into (A.14), and upon using

$$
1+\tan ^{2}\left(\theta_{0}\right)^{2}=\sec ^{2}\left(\theta_{0}\right)^{2}
$$

we have

$$
\begin{aligned}
y=1 & \pm h \tan \left(\psi_{1}\right)\left(1+\tan (\theta) \tan \left(\theta_{0}\right)\right) \cos \left(\theta_{0}\right) \\
& \cdot \sqrt{1-\left\{\frac{\tan \left(\theta-\theta_{0}\right)}{\tan \left(\theta_{1}\right)\left(1+\tan (\theta) \tan \left(\theta_{0}\right)\right)}\right\}^{2}} \cdot
\end{aligned}
$$

To measure $x$ taking point $K$ as a reference, the distance $K A=$ $h \tan \left(\theta_{0}-\theta_{1}\right)$ should be added to (A.1) so the new $x$ becomes

$$
x=h \tan (\theta) .
$$

Substituting this last equation into (A.17) gives the locus of the beam on the ground as

$$
\begin{gathered}
y= \pm h \tan \left(\psi_{1}\right) \cos \left(\theta_{0}\right)\left(1+\frac{x}{h} \tan \left(\theta_{0}\right)\right) \\
\cdot \sqrt{1-\left(\frac{1}{\tan \left(\theta_{1}\right)}\right)^{2}\left\{\frac{\frac{x}{h}-\tan \left(\theta_{0}\right)}{1+\frac{x}{h} \tan \left(\theta_{0}\right)}\right\}^{2}}
\end{gathered}
$$

where $h \tan \left(\theta_{0}-\theta_{1}\right) \leq x \leq h \tan \left(\theta_{0}+\theta_{1}\right)$. If a point $(x, y)$ is rotated by an angle $\phi_{0}$, the new coordinates will be

$$
x^{\prime}=x \cos \left(\phi_{0}\right)-y \sin \left(\phi_{0}\right)
$$

and

$$
y^{\prime}=y \cos \left(\phi_{0}\right)+x \sin \left(\phi_{0}\right)
$$

\section{ACKNOWLEDGMENT}

The authors acknowledge the discussions with Dr. D. Nunn, D. Stewart, and S. L. Dhomeja from the Department of Electronics and Computer Science, University of Southampton, U.K. They are also grateful to B. Sultan, Department of
Civil Engineering, University of Southampton, for his help in deriving the footprint equations.

\section{REFERENCES}

[1] R. Steele, Communication After AD 2000, ch. Mobile Communications in the 21st Century. New York: Chapman Hall, 1993.

[2] —_, "An update on personal communications (Guest Editorial)," IEEE Commun. Mag., vol. 30, pp. 30-31, Dec. 1992.

[3] Y. C. Lee and M. Rothblatt, "Stratospheric telecommunications service: An opportunity to close the information gap," ITU News, no. 3, pp. 25-29, 1997.

[4] B. El-Jabu and R. Steele, "Effect of positional instability of an aerial platform on its CDMA performance," in Proc. VTC'99 Fall, Amsterdam, The Netherlands, Sept. 1999.

[5] C. A. Balanis, Antenna Theory: Analysis and Design. New York: Harper Row, 1982.

[6] R. E. Collin, Antenna and Radio-wave Propagation. New York: McGraw-Hill, 1985.

[7] G. M. Djuknic, J. Freidenfelds, and Y. Okunev, "Establishing wireless communications services via high-altitude aeronautical platforms: A concept whose time has come," IEEE Commun. Mag., pp. 128-135, Sept. 1997.

[8] ETSI/SMG/SMG, The ETSU UMTS Terrestrial Radio Access (UTRA) ITU-R RTT candidate submission, , 1998.

[9] W. C. Y. Lee, "Overview of cellular CDMA," IEEE Trans. Veh.Technol., vol. 40, pp. 291-302, May 1991.

[10] R. Steele, "Communications++: Do we know what we are creating?," in Proc. EPMCC'97, VDE-VERLAG GMBH, Berlin, Germany, Sept. 1997, pp. 19-23.

[11] B. Sklar, Digital Communications: Fundamentals and Applications. Englewood Cliffs, NJ: Prentice-Hall, 1988.

Bashir El-Jabu was born in Misurata, Libya, in 1957. He received the B.Sc. degree in electrical engineering from Al-Fateh University, Libya, in 1980, the M.Sc. degree in electrical engineering from Queens University, Canada, in 1986, and the Ph.D. degree in electronics from the University of Southampton, Southampton, U.K., in 1999.

$\mathrm{He}$ is an Assistant Professor in the Electrical Engineering Department, Al-Fateh University, Libya, and Head of Scientific Department at the Higher Industrial Institute, Misurata, Libya. His current research interests are mobile communications and signal processing. He has published many papers in the field of communications, signal processing, and education.

Raymond Steele (SM'80-F'96) is Chairman of Multiple Access Communications Ltd., a company he formed in 1986 that is concerned with consultancy and products in digital mobile radio systems. His former posts were as Head of the Communications Research Group in the Department of Electronics and Computer Science, University of Southampton; a Research Worker with Bell Laboratories; a Research and Development Engineer with the Marconi Company, Cossor Radar \& Electronics, and E. K. Cole Ltd.; and a Senior Lecturer at the University of Loughborough. He is the author of Delta Modulation Systems (New York: Halsted, 1975), editor of Mobile Radio Communications (New York: IEEE Press and Wiley, 1992), coauthor of Source-Matched Mobile Communications (New York: IEEE Press and Wiley, 1995), and author of more than 200 technical publications. He has been a Conference and Session Organizer of numerous international conferences and a Keynote Speaker at many international meetings. He is a member of the Advisory Board of the International Journal of Wireless Information Networks.

Prof. Steele is a Fellow of The Royal Academy of Engineering and the IEE, U.K. He is a Member of the IEEE Avant Garde and is an IEEE Distinguished Speaker. He is a Member of the Advisory Board of IEEE Personal Communications (the magazine of nomadic communications and computing). He and his coauthors received the Marconi Premium in 1979 and 1989 and the Bell System Technical Journal's award for the Best Mathematics, Communications, Techniques, Computing and Software, and Social Sciences Paper in 1981. 